\title{
THE MAGNETOHYDRODYNAMIC WAKE OF THE MOON
}

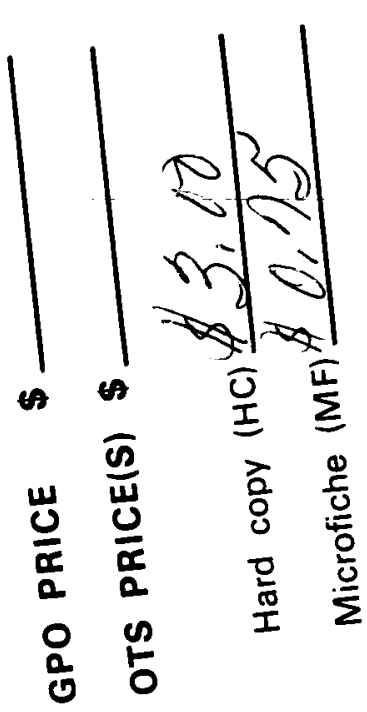

BY

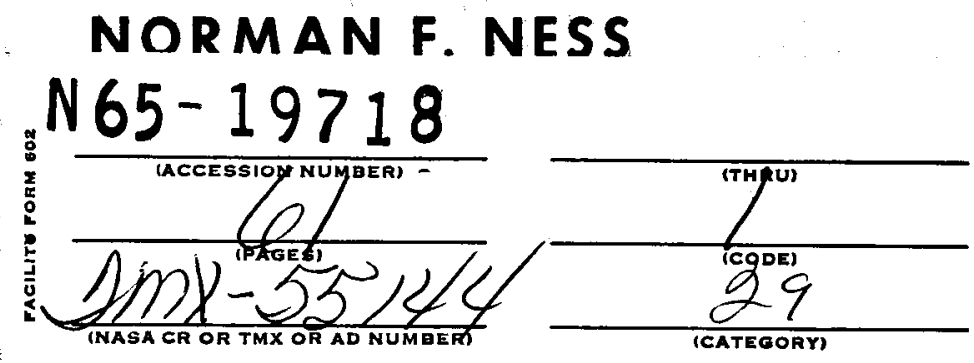

OCTOBER 1964

NASA

GODDARD SPACE FLIGHT CENTER GREENBELT, MD. 
The Magnetohydrodynamic Wake of the Moon

\author{
Norman F。 Ness \\ NASA-Goddard Space Flight Center \\ Greenbelt, $\mathbf{H d}$.
}

To be submitted to 


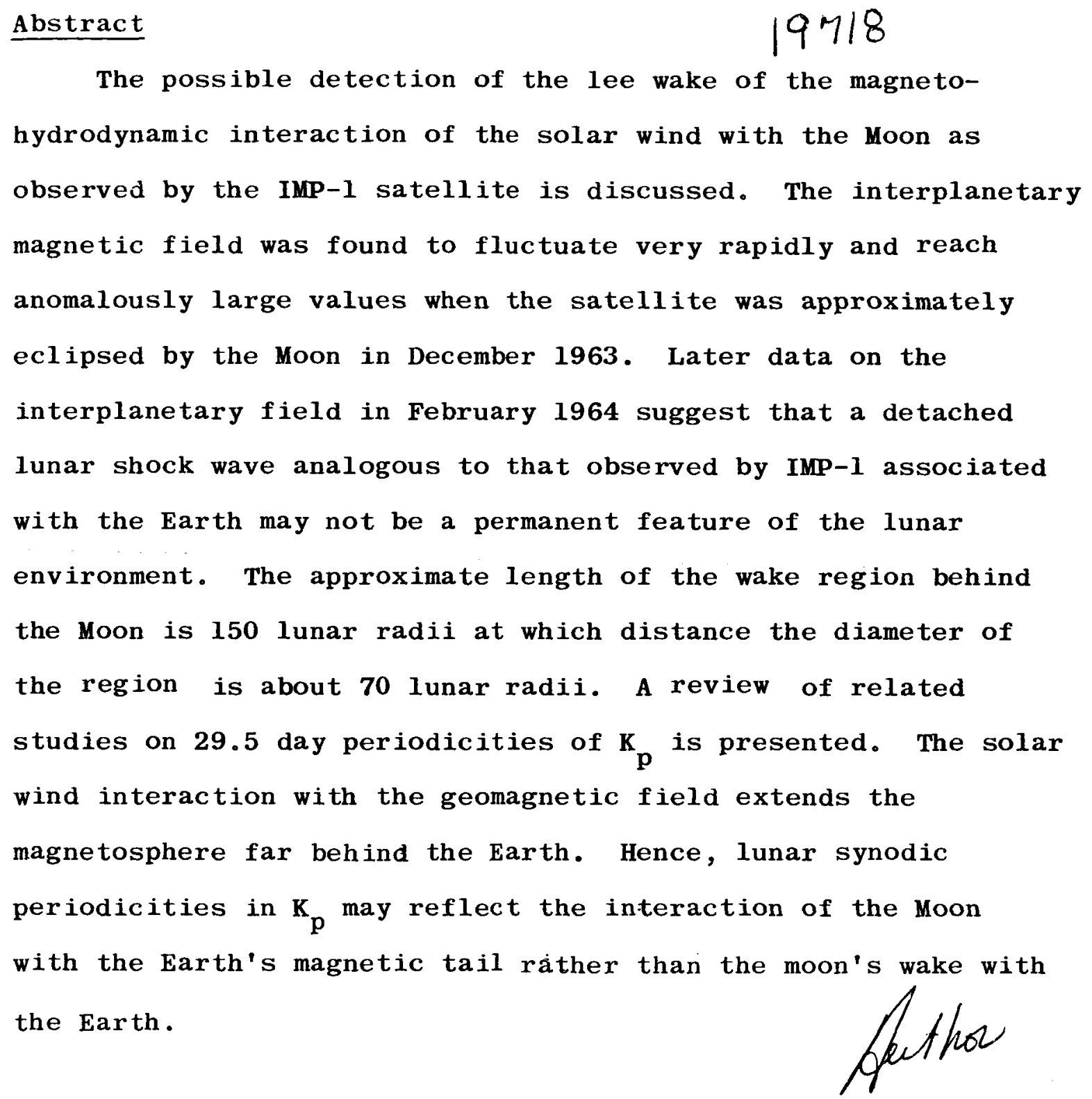


Table of Contents

Page

$1.0 \quad$ Introduction $\ldots \ldots \ldots \ldots \ldots \ldots \ldots \ldots \ldots \ldots \ldots \ldots \ldots$

2.0 Magnetic field of the Moon. . . . . . . . . . . . . . . . . 3

3.0 Lunar Shock Wave... . . . . . . . . . . . . . . . . . . 6

4.0 Observations of the Lunar Magnetohydrodynamic Wake. . . 10

5.0 Fluid Dynamic Considerations. . . . . . . . . . . . . . 18

$6.0 \mathrm{~K}_{\mathrm{P}}$ and Related Studies . . . . . . . . . . . . . . . . . 24

7.0 Summary and Conclusions . . . . . . . . . . . . . . . 27

Acknowledgements. . . . . . . . . . . . . . . . 29

References

List of Figures

Figures $1-17$ 
The Magnetohydrodynamic Wake of the Moon

Norman F. Ness

$\underline{1.0 \quad \text { Introduction }}$

Lunar associated terrestrial phenomena have been studied extensively for many years in a wide range of scientific disciplines including geomagnetism (Chapman and Bartels, 1940). The principal geophysical effects which have been observed are due primarily to the direct or indirect ilfluence of the Moon's gravitational field. The rotation of the Earth coupled with the elliptic orbit of the Moon introduces characteristic time modulations and fluctuations of geophysical phenomena with frequencies which can be accurately ascribed to a lunar gravitational influence. The various "tidal" phenomena with semi-diurnal to monthly periods occurring in both the solid and fluid Earth contain important lunar constituents (Melchior, 1957; Doodson, 1958, Siebert, 1960). Recently attention has been directed to a study of the expansion of the solar corona into interplanetary space. This can lead to a somewhat different and more complex lunar as well as solar influence on the inmediate terrestrial environment。Fejer (1964) has suggested that at least a portion of the classical "tidal" variations in geomagnetism may be directly related to this phenomenon. 
Subsequent to the early suggestion by Biermann (1951). that an adequate explanation of cometary tail structures required a substantial and continuous solar corpuscular flux, Parker $(1958,1960)$ developed the hydrodynamic theory of the coronal expansion, referring to this plasma flow as the "solar wind". Direct measurements by satellite and space probes since 1959 have confirmed experimentally the existence of a solar plasma flowing approximately radially from the Sun with a positive ion flux between $10^{7}$ to $10^{9}$ particles $/ \mathrm{cm}^{2} / \mathrm{sec}$ and energies between $200 \mathrm{ev}$ to $10 \mathrm{Kev}$ (Gringauz, 1961; Bonetti et al, 1963; Snyder and Neugebauer, 1964; Bridge et al, 1964). A direct consequence of the radial solar wind velocity, the highly ionized coronal gases, and the rotation of the Sun is that the photospheric magnetic fields are extended in the plane of the ecliptic and twisted into the form of an Archimedes spiral (Parker, 1963). Accurate measurements of the interplanetary magnetic $f$ ield have recently been made on the IMP-1 satellite (Ness et al, 1964) confirming certain of the theoretical predictions on the direction of the field and determining the average magnitude to be close to 5 gammas (Ness and Wilcox, 1964)。

The interaction of the solar wind with planetary objects is an important factor in determining their space environment. The principal result of the solar wind impacting a planetary magnetic field is to confine the field to a region of space surrounding the planet. The existence of a quasi-static Chapman-Ferraro cavity (reviewed by Chapran (1963)) which excludes the direct 
penetration of solar plasma into the geomagnetic field has been demonstrated by the recent satellite experiments of Bonetti et al (1963), Heppner et al (1963), Cahill and Amazeen (1963), Freeman et al (1963), Ness et al (1964), Anderson et al (1964), Bridge et al (1964), and Freeman (1964)。An important feature of the interplanetary medium, with respect to its interaction with planetary objects, is that it is a magnetized plasma and thus supports magnetohydrodynamic wave propagation. In this sense the plasma flow is super-Alfvénic since the velocity of magnetohydrodynamic wave propagation is less than $90 \mathrm{~km} / \mathrm{sec}$ while the solar plasma velocity is between $300-900 \mathrm{~km} / \mathrm{sec}$. This leads to Alfvén Mach numbers usually greater than 4 .

This paper is concerned with an expanded discussion of possible direct experimental evidence of the magnetodydrodynamic interaction of the solar wind with the Moon as obtained by the IMP-1 magnetic field experiment (Ness et al, 1964)。 During the month of December 1963 the IMP-1 satellite appears to have been located within the downstream wake region of the Moon as it interacts with the solar wind. This interpretation of the data as well as the data itself will be discussed in the following sections. 2.0 Magnetic Field of the Moon

The existence or absence of an intrinsic lunar magnetic field is important in the development of adequate theories to explain planetary magnetism and the origin of the solar system. The presently accepted theory for the origin of the geomagnetic 
field is based upon a fluid core sustaining a regenerative dynamo system of electrical currents (Elsasser, 1950; Inglis, 1955). The possibility of a lunar magnetic field maintained by such fluid motion has been theoretically investigated by Zharkov and Ulinich (1960) concluding that surface fields of 0.1 to 1.0 gauss are possible. The internal constitution of the Moon is not well known (MacDonald, 1961) but the possibility of a dense fluid core analogous to the Earth's seems highly unlikely. Another possibility is that sometime in the ancient past the Moon cooled down through its Curie point while in the presence of an external magnetic field thus acquiring thermoremanent magnetization. This latter suggestion requires that the Moon was at one time nearly entirely molten whether it accreted from a large number of smaller masses or was formed from one large mass. At the present time this hypothesis cannot be conclusively argued (Levin (1960), MacDonald (1963)).

The only direct evidence of the state of a lunar magnetic field has been provided by the Soviet space probe Lunik II (Dolginov et al, 1961). Measurements with a sensitivity of about 30 gammas were performed along an impact trajectory to within a distance of $55 \mathrm{~km}$ from the lunar surface. No lunar magnetic field larger than the noise level was detected. The conclusion reached by these authors on the basis of a simple dipole model of the lunar magnetic field was that the effective lunar magnetic moment must be less than $10^{-4}$ that of the Earth's. As pointed out by Vestine (1957) and Neugebauer (1960) however, 
the solar wind is sufficiently strong to confine the magnetic field of the Moon to a region very close to or even below its surface. In the latter case the solar wind directly impacts the lunar surface. On the assumption that

(1) the Moon possesses a dipolar magnetic field with equatorial field strength $=\mathrm{B}_{\mathrm{O}}$,

(2) the dipole axis is perpendicular to the solar wind velocity and

(3) the solar plasma flows on around to the back side of the Moon rather than to be specularly reflected then the radius of the lunar magnetic cavity boundary at the subsolar or stagnation point is given by :

$$
R_{c}=R_{m}\left[\frac{B_{0}^{2}}{2 \pi m n v_{s}^{2}}\right]^{1 / 6}(2 \circ 1)
$$

where $\quad \mathrm{V}_{\mathrm{S}}=$ velocity of solar wind $(\mathrm{Km} / \mathrm{sec})$

$$
\mathrm{n}=\text { proton density }\left(\mathrm{p} / \mathrm{cm}^{3}\right)
$$$$
\mathrm{m}=\text { proton mass (gms) }
$$

This is illustrated in Figure 1 and clearly shows that for the observed values of the solar wind any such lunar magnetic field will be compressed rather close to the surface of the Moon.

Gold (1964) has suggested an origin of a lunar field which incorporates the interaction of the magnetized solar plasma with the lunar body. The finite electrical conductivity of the Moon provides a mechanism for the trapping of the interplanetary 
magnetic field. The model proposed has not been quantitatively developed. It depends critically upon the magnetic Reynolds number of the Moon, requiring that the interplanetary field be obstructed in its motion through the lunar body so that lines of force are accumulated and compressed on the sunlight hemisphere. This must occur for a time interval sufficiently long that a quasi-static field configuration can develop in spite of the rotation of the Moon and the observed variability of the direction of the interplanetary magnetic field.

3.0 Lunar Shock Wave

Tine presence or absence of a lunar magnetic field is important in determing the detailed characteristics of the solar wind interaction with the Moon. Far downstream it is not as clear how important any lunar field may be in determining the characteristics of the resultant interactions in the solar wind as it resumes a free stream flow. The overall situation may well resemble more closely the interaction of the solar wind with comets (Marochnik, 1963) rather than its interaction with the geomagnetic field. For the present study, the most pertinent feature of the solar wind interaction is the distance behind the Moon to which observable effects of such magnetohydrodynamic phenomena would persist. Various authors (Beard, 1960; Johnson, 1960; Piddington, 1960; Harrison, 1963; Lees, 1964; Axford et al, 1964 and Dessler, 1964) have presented qualitative arguments on the length of the geomagnetic "tail" behind the Earth. This region of the magnetosphere has yet to be experimentally probed and existing theoretical treatments are limited in their scope. 
The best efforts thus far have utilized the analogy with supersonic gas dynamics representing the solar wind as a viscous fluid and translating the particle characteristics of the plasma to a continuum property of the fluid.

The suggestion that a detached collisionless magnetohydrodynamic shock wave encloses the Earth's magnetosphere has been presented by Axford (1962) and Kellogg (1962) among others. Spreiter and Jones (1963) have applied classical supersonic flow theory to determine the location and geometry of the shock wave surface. The agreement between theory and observation is very good, requiring only a minor modification in the choice of equivalent specific heat ratio used in the fluid dynamic analogy to yield improved comparisons with the observations. The most critical point in these studies being the ratio at the stagnation point of the shock wave radius to the geomagnetic cavity radius. The success of this similitude argument has been verified experimentally only near the stagnation point of the solar wind interaction with the Earth's magnetic field. However this suggests its consideration for conditions far from the stagnation point, i.e. the tail region of the solar wind interaction with the geomagnetic field. This has been done by Lees (1964) who showed that a secondary shock or tail shock would develop in the wake of the plasma flow around the earth. Data obtained by the IMP-l satellite which have yet to be analyzed will partially answer these important questions about the magnetic field topology within the geomagnetic tail. 
In the absence of more definite knowledge about a lunar magnetic field the argument for a lunar shock wave is not on as firm a basis as in the case of the Earth. In the relatively weak interplanetary magnetic field the radius of gyration of a solar wind proton is comparable to the lunar radius. Table I presents representative values for the proton Larmor radius as a function of field strength and plasma energy (or velocity).

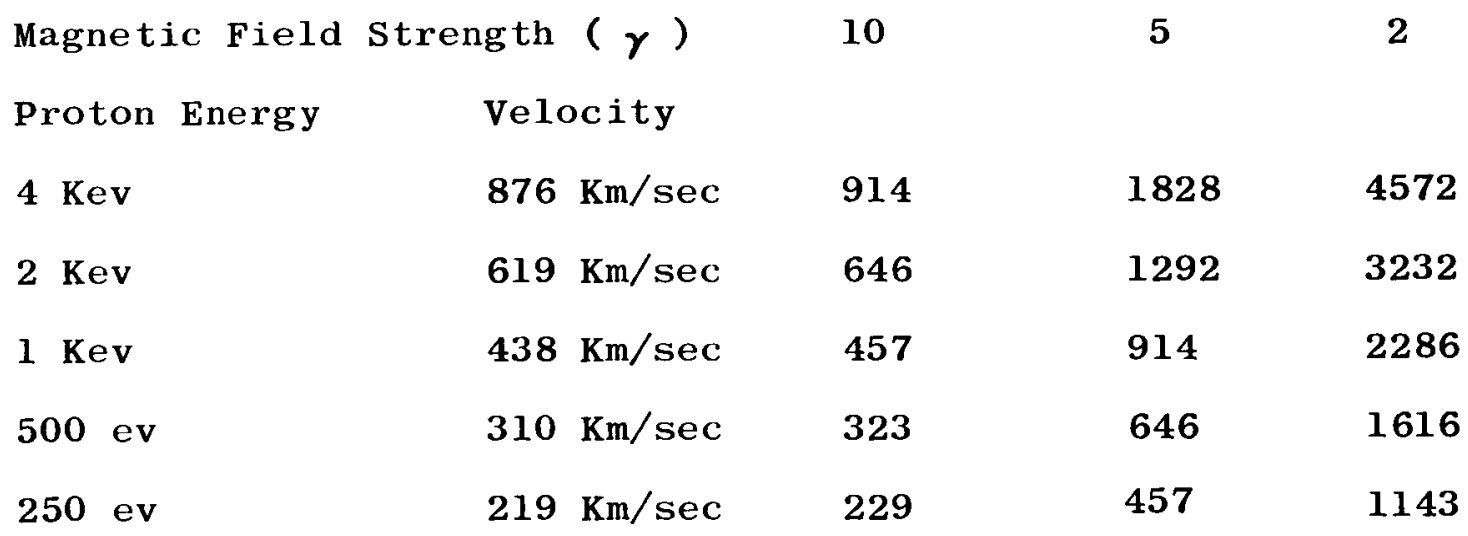

TABLE I

Proton Larmor radius (in Kilometers) as function of particle Energy (or velocity) and magnetic field strength (gammas).

The Larmor radius is equal to or greater than the stand off distance of $550 \mathrm{~km}$. of the shock wave at the stagnation point that is obtained using the value of $R_{\mathrm{S}} / R_{\mathbf{C}} \stackrel{\ominus}{=} .31$ as measured in the case $o_{i}$ the Earth (Ness et al, 1964). For the case of a spher'e Kellogg (1962) using Hida's (1953) analysis of a shock surrounding a spherical object determined the stand of $f$ ratio 
to be approximately 1.25 for Mach values greater than 4 . It may well be that in the case of the moon a collisionless shock wave analogous to the Earth's may not develop at all times, if indeed at all. Whether or not such a shock develops depends very critically upon the physical parameters in the interplanetary medium and the state of the lunar magnetic field. This point has also been discussed by Gold (1964) with regard to the direct penetration of the solar plasma into the lunar body。

The detailed characteristic of the lunar surface and atmosphere are strongly dependent upon whether or not the surface is shielded from direct impact of the solar wind by either a lunar field or a shock wave. Studies on these and related lunar surface characteristics have been performed by Bernstein et al (1963), Wehner et al (1963), Hapke (1964), Hinton and Taeusch (1964) and Kopal and Rackham (1964). 4.0 Observations of a Lunar Magnetohydrodynamic Wake

The IMP-1 magnetic field experiment has been described in an earlier publication (Ness et al, 1964) which should be consulted for a detailed description of the satellite, its orbit, the instrumentation and telemetry system and the general characteristics of the interplanetary magnetic field. In addition the interaction of the solar wind with the geomagnetic field is discussed. In the following presentation the positions of the satellite, earth and moon are initially presented in the solar ecliptic coordinate system defined as : $x_{s e}$ is the axis from the Earth to the Sun, $\mathrm{Z}_{\mathrm{se}}$ is the axis normal to the ecliptic 
plane and in the same sense as the Earth's angular momentum and $\mathrm{Y}_{\text {se }}$ which forms a right handed coordinate system. All distances are given in units of an Earth radius $\left(R_{e}=6378.4 \mathrm{Km}\right)$ although in the final interpretation of the data a translation will be made to a lunar associated coordinate system using a scale in Moon radii $\left(R_{m}=1738 \mathrm{Km}\right)$ 。

The positions of the Moon and IMP-1 during December 1963 are shown in Figure 2 as projected on the plane of the ecliptic。 The solar wind direction has been adjusted to appear to come from $5^{\circ}$ west of the Sun in order to represent the aberration effect due to the heliocentric motion of the Earth. The orbital velocity of the Earth is $30 \mathrm{Km} / \mathrm{sec}$ and for a solar wind velocity of approximately $330 \mathrm{Km} / \mathrm{sec}$ the angular displacement is $5^{\circ}$. Ness and Wilcox (1964) have determined from a correlation of the solar photospheric magnetic field and the interplanetary field as measured on IMP-1 that the average solar wind velocity was approximately $385 \mathrm{Km} / \mathrm{sec}$ during the time interval November 27, 1963 to February 19, 1964. Thus the correction for a $5^{\circ}$ aberration is slightly larger than would be justified on the basis of experimental data. The projected traces of the average position of the magnetopause and shock wave boundaries are also included in the figure in order to show when the satellite was outside of these boundaries and within the relatively undisturbed interplanetary medium. It is seen that on December 14 the satellite was close to apogee and approximately in line with the Moon with respect to the propagation of the solar wind. 
This is a measure only in the XY plane however, and does not include the distance of the satellite either above or below the Sun-Moon line.

In Figure 3 the positions of the satellite and the Moon during the same time interval are shown projected on to the plane perpendicular to the $\mathrm{x}_{\text {se }}$ axis. The satellite was several Earth radii below the Moon during the entire period of interest, December 14-15. The location of the satellite with respect to the Moon as a function of time is summarized in Figure 4. The two angles $\lambda_{\mathrm{xy}}$ and $\lambda_{\mathrm{yz}}$ are introduced as a measure of the apparent angular displacement of the Moon with respect to the satellite as projected onto the XY and YZ planes respectively. The quantity $D_{y z}$ measures the projected distance in the $\mathrm{YZ}$ plane, $\mathrm{D}_{\mathrm{Sm}}$ measures the distance from the satellite to the Moon and $\mathrm{D}_{\mathrm{y}}{ }^{\prime} \mathrm{z}$ measures the distance projected onto a $\mathrm{YZ}$ plane which has been rotated by $5^{\circ}$ West so as to be perpendicular to the aberrated solar wind. These parameters show that on December 14 at approximately 0915 the satellite was close to being immediately behind the Moon. At this time the satellite was $37.8 \mathrm{R}_{\mathrm{e}}$ from the Moon and $8.7 \mathrm{R}_{\mathrm{e}}$ below the Sun-Moon line. The aberration of the solar wind by $5^{\circ}$ leads to a change in the alignment of the satellite with regard to the Moon and solar wind as shown by the dashed curve in Figure 4. The time at which the satellite is closest to being aligned with the Moon-Sun direction occurs several hours in advance of the non-aberrated case although the distance is approximately the same. On the basis of these data the most probable time for the observation of solar wind interactions with the lunar body would be centered around 0900 
December 14, on the assumption that the lunar wake extended in the general flow direction behind the Moon.

The 5.46 minute averages of the magnetic field obtained by this experiment for the time interval 1600 December 13 to 1600 December 15 are shown in Figures 5 and 6 . The format for the presentation is identical to that used by Ness et al (1964). The two angles $\theta$ and $\phi$ measured the latitude and longitude respectively of the magnetic field vector in the solar ecliptic coordinate system. In figure 5 the first 9 hours of data show the characteristic behavior of the interplanetary magnetic field with a stable orientation and magnitude. At this time the magnetic field was directed approximately in the plane of the ecliptic $\left(\theta=0 \pm 30^{\circ}\right)$ and pointed back toward the sun along the Archimedian spiral angle predicted for the interplanetary field $\left(\phi=315^{\circ} \pm 20^{\circ}\right)$. During this time the satellite is close to apogee, (geocentric distance $=31.7 R_{e}$ ) and far removed from any direct effects of the solar wind interaction with the Earth. As time progresses the field becomes variable in both magnitude and direction. The onset of this turbulence occurs at approximately 0130 December 14, and persists until 0900 December 15. Midway in this time interval the field magnitude reaches the anomalously large value of about 15 gammas for more than 3 hours, from 18002100 December 14, while the turbulent characteristics of the field as measured by the angle $\phi$ noticeably decreases. Following 0900 December 15 the magnetic field again resumes its characteristic stable configuration although it is not as stable as it was prior to these events. 
Superimposed on the time plots are specific values of parameters related to the position of the satellite with respect to the Moon. It should be noted that when the satellite is closest to being in line $\left(\lambda_{\mathrm{xy}}=0\right)$ the distance $\mathrm{D}_{\mathrm{yz}}$ is not necessarily at a minimum. Indeed the minimum value of $\mathrm{D}_{\mathrm{yz}}$ occurs at 1830 December 14 and is approximately the same time as the midpoint of the time interval during which the turbulent magnetic field observations were made. The closest aberrated distance, $D_{y^{\prime} z}=9.3 R_{e}$, occurs at 0840 . The characteristics of the magnetic field during this time interval are unlike those during other occasions on which the satellite was in the interplanetary medium. The turbulence and large field values, as well as the orientation during the time of large field magnitudes suggest that the IMP-1 satellite passed through the turbulent lee wake of the Moon in the solar wind. The strong fields observed correspond to the magnetosphere of the Moon and the turbulent fields to the transition region between the lunar magnetosphere and the undisturbed interplanetary medium.

In Figure 7 the positions of IMP-1 and the Moon during January 1964 are shown as projected onto the ecliptic plane. This presentation is identical to that for Dəcember as shown in Figure 2. Here, however, the relative positions of the satellite and the Moon are seen to be quite different. The time at which IMP-1 is roughly aligned with the Moon occurs at 0730 January 13 but at this time the satellite is enclosed within the Earth's shock wave. Figure 8 presents the relative positions of IMP-1 and the Moon as projected onto the YZ plane. The 
satellite is about in the same position relative to the plane of the ecliptic as in December but the Moon is considerably lower. A summary of the relevant position parameters related to the relative positions of IMP-1 and the Moon is given in Figure 9. The format is identical to that of Figure 4 and illustrates that during the 13 th orbit when the satellite was in the lee of the Moon it was also within the region of space surrounding the Earth enclosed by the shock wave surface. The time at which the Earth's shock wave was traversed by the satellite is indicated by the solid vertical line. It occurred several hours after the satellite was aligned with the Moon and the Sun. The situation with respect to the aberrated solar wind direction does not favor the observation of a lunar wake since the satellite is seen to spend a longer time within the region of space associated with the solar wind interaction with the geomagnetic field. Thus the satellite motion in the month of January 1964, was such as to preclude being ill a favorable position with respect to observation of the lunar wake. In spite of this the interplanetary magnetic field data for January 13 and 14 has been inspected in detail since the December observation extended over a time period of more than one day. It has not disclosed any characteristics which are suggestive of a lunar wake, either in turbulence or field magnitude.

The last opportunity for observation of the lunar wake with IMPl occurred in February 1964 during orbit 20. On the succeeding opportunities for wake observations during the next six months, 
the IMP-l satellite was enclosed completely within the interaction region of the solar wind with the geomagnetic field. The steady precessional motion of the IMP-1 orbit in the solar ecliptic coordinate syste:n is evident by comparing Figure 10 for February 1964 with that of Figure 2 for December 1963. The relative positions of IMP-1 and the Moon in February are such that they are approximately aligned at 0020 February 11 . The position of these two objects in February projected onto the $\mathrm{YZ}$ plane is shown in Figure 11. Both the elongation of the IMP-1 orbit relative to Figures 3 and 8 and the location of the Moon below the ecliptic plane are a result of the heliocentric motion of the Earth about the Sun. It can be seen from this figure, however, that the two objects are very close to being in precise alignment. Figure 12 summarizes the relative positions of IMP-1 and the Moon as a function of time in a format identical to that used for Figures 4 and 9 . The distance from the satellite to the Moon is seen to have increased now to $57.9 \mathrm{R}_{\mathrm{e}}$ while the minimum value for the distance in the $\mathrm{YZ}$ plane is $1.7 \mathrm{R}_{\mathrm{e}}$. For the aberrated solar wind the closest distance $\mathrm{D}_{\mathrm{y}^{\prime} \mathrm{z}}$ of $3.7 \mathrm{R}_{\mathrm{e}}$ occurs at 1640 February 10 。

The magnetic field data for the time interval 0430 February 10 to 0430 February 12 is shown in Figure 13 and 14. The data in Figure 13 illustrates the outbound traversal of the shock wave associated with the Earth's magnetosphere at a distance of $30.3 \mathrm{R}_{\mathrm{e}}$ at 0958 February 10. The characteristic of large turbulent fields in the transition region is clearly evidenced. Also 
shown and to be discussed in a future separate publication on the magnetic field characteristics in the transition region is the compressed and reasonably well aligned magnetic field such as were first observed by Explorer X (Heppner et al, 1963). The abruptness of the shock boundary, as shown by the variance plot, is a persistent feature of the shock phenomena in which the thickness is small and comparable with the ion gyro-radius. Subsequent to the outbound shock wave traversal the magnetic field in interplanetary space is somewhat variable in direction as measured by $\theta$ and $\phi$ but is not turbulent in the same sense as in the transition region as measured by the variance characteristics. However, during the time interval 0400 to 1200 February 11 there is a small increase above the noise level in the turbulence as measured by the variance. At the same time the variability between successive values of $\theta$ and $\phi$ is increased. However, during the time interval while the variance is higher than the noise level, the field is at its lowest value, less than 3 gammas, during this sample of the interplanetary medium on orbit No. 20 .

Figure 14 contains the remainder of the interplanetary magnetic field data observed on orbit 20 and also illustrates the inbound traversal of the Earth's shock wave by the IMP-I satellite. Again the characteristics of the transition region on the inbound pass are quite similar to those observed on the outbound traversal although they occur at a different geocentric distance, $23.6 \mathrm{R}_{e}$, with a suggestion of a "precursor" at $24.3 \mathrm{R}_{e^{\circ}}$ 
The nonspherical symmetry of the shock wave surface enclosing the Earth coupled with the fact that the satellite traverses these boundaries at considerably different satellite-earth-sun angles on successive transits leads to these differences. Throughout the time interval during which the interplanetary medium was sampled, features similar to the December 1963 observation can not be seen. At this time it is suggested that the February 1964 data show characteristics which are similar in some respects but are not as conclusive an evidence of a lunar wake as observed in December 1963. $\underline{5} .0 \quad$ Fluid Dynamic Considerations

The classical magnetohydrodynamic problem of the interaction of a spherical object with a conducting fluid in the presence of a magnetic field has been treated by Stewartson (1956), Imai (1960) and Ludford and Murray (1960) among others. For the case of either compressible or incompressible fluid flow a downstream wake always develops for large or small magnetic Reynolds numbers as long as the magnetic field strength is small (Sears (1960)). This is measured by the ratio of free stream velocity $\left(v_{s}\right)$ to the pure Alfven velocity $\left(v_{a}\right)$ or the geometric mean $\left(V_{a}{ }^{*}\right)$ of the Alfven and sound velocities. This ratio, Vs/Va or $\mathrm{Vs} / \mathrm{V}_{\mathrm{a}} *$, must be greater than unity in order that a lee wake develop and effectively determines whether or not the presence of the body in the flow field can be communicated upstream by magnetohydrodynamic wave propagation. A major limitation in the quantitative use of the presently available analytical treatments 
is that they employ restrictive assumptions which are not justified in the case of the interaction of the solar wind with the Moon. In general these include one or all of the following: infinitely conducting fluid, magnetic fields aligned with the flow velocity, nonconducting or infinitely conducting body, and collision mean free paths much smaller than other lengths characteristic of the flow. The case of the Moon in the solar wind satisfies few of these assumptions and thus direct comparisons are not possible. Further development of analytical studies on collisionless magnetohydrodynamic shocks (see review by Kantrowitz and Petschek (1964)) is required for application to the present problem.

In the case of the interaction of the solar wind with the geomagnetic field rather successful results, at least near the stagnation point, have been obtained through use of a pure fluid dynamic analogy. A general review of the use of the aerodynamic similitude and its application to the solar wind interaction with the Earth's magnetic field has been given by Levy et al (1964) and Lees (1964)。On this basis an important aspect of the interpretation of the magnetic field data on IMP-1 is that the free stream Mach number can provide a measure of the angular width of the shock surface associated with the interaction. (Michel (1964) has treated this problem assuming complete magnetohydrodynamic flow of the solar wind.)

However, the detailed characteristics within the interaction region and more importantly the wake region may not be completely specified. The Mach number in the case of the solar wind is 
measured by the ratio of the plasma bulk velocity $\left(\mathrm{v}_{\mathrm{S}}\right)$ to the magnetohydrodynamic wave phase velocity $\left(\mathrm{v}_{\mathrm{a}}\right)$. In the interplanetary medium the velocity $v_{a}$ is approximately given by the Alfvén velocity so that the Mach number is given by

$$
M_{a}=\frac{V_{s}}{V_{a}}=\frac{V_{s} \sqrt{4 \pi m n}}{B}
$$

where $B$ is the strength of the interplanetary field.

A plot of the Alfven velocity as a function of the plasma density and magnetic field strength is shown in Figure 15. From classical supersonic flow (Hayes and Probstein, (1959)) the Mach angle $\beta$ associated with a specific Mach number is given by

$$
\beta=\operatorname{SIN}^{-1}\left(\frac{1}{M_{a}}\right)
$$

and represents the asymptotic direction of the shock surfaces far downstream from the obstruction. The Mach angle is plotted in Figure 16 as a function of the Mach number.

The average magnetic field in the interplanetary medium has been deterinined by the IMP-1 measurements to be 5.0 gammas. The average solar wind velocity has been estimated to be approximately $385 \mathrm{Km}$. Using these average values for the magnetic field and solar wind velocity and assuming a plasma density between 1.5 to $35 \mathrm{p} / \mathrm{cm}^{3}$ yields a Mach number between 4 and 20 . 
For these two values of Mach number the limits on the Mach angle are $14.5^{\circ}$ and $2.8^{\circ}$ respectively。

Obayashi (1964), in a review of previous satellite measurements related to the solar wind interaction, has adapted classical hydrodynamic equations for approximating the geometry of the shock surface surrounding a spherical object. Defining :

$$
\begin{aligned}
& \mathrm{R}_{\mathrm{S}}=\text { the radial distance to the shock from the center } \\
& \text { of the sphere at the stagnation point } \\
& \mathrm{R}=\text { the radial distance to the shock surface } \\
& \phi=\text { the angle between } R \text { and } R_{S} \text {, then he deduces that: } \\
& R=R_{S}\left[\frac{1+\operatorname{SEC} \beta}{1+\operatorname{SEC} \beta \operatorname{COS} \phi}\right]
\end{aligned}
$$

A translation of the position of the IMP-1 satellite to a lunar set of coordinates has been made for the pertinent time periods in December 1963 and February 1964. The position of the satellite in these lunar associated coordinates is shown in Figure 17 with the abscissa being given by $D_{x y}$ or $D_{x y}$ ' where

$$
\begin{aligned}
& D_{x y}=\sqrt{\left(D_{s m}\right)^{2}-\left(D_{y z}\right)^{2}} \\
& D_{x y^{\prime}}=\sqrt{\left(D_{s m}\right)^{2}-\left(D_{y_{z}^{\prime}}\right)^{2}}
\end{aligned}
$$


and the ordinate by $D_{y z}$ or $D_{y^{8} z}$. This presentation essentially assumes that far down stream from the Moon the lunar wake region is cylindrically symmetrical about the solar wind flow direction. Superimposed on these trajectory positions are the two shock surfaces corresponding to Mach numbers 4 and 20。 These surfaces assume that the lunar magnetic field strength is inherent1y less than a few hundred gammas and that the shock stand off ratio $R_{S} / R_{m}=1.25$. It is seen that if the flow is such that the Mach number is less than about 10 then the position of the satellite in December 1963 is well within the shock region of the lunar wake. If the Mach number of the flow is less than about 40 then the satellite is within the shock for February 1964.

The detection of a lunar wake is definitely consistent with supersonic flow theory for the orbit No. 5 observation and with the orbit 20 observation. However, even though the wake was possibly observed in December 1963, the interaction of the solar wind with the Moon is probably so variable that appropriate interplanetary conditions in the vicinity of the Moon are required in order to initiate the formation of a collisionless magnetohydrodynamic shock. Indeed using the average velocity of $385 \mathrm{~km} / \mathrm{sec}$ and the average field strength of 5 gammas leads to a proton Larmor radius of $830 \mathrm{Km}$. This implies that on average the shock wave radius assumed for the Moon, $2173 \mathrm{Km}$, is only 2.6 times this critical value. The stand off distance is about $430 \mathrm{Km}$ so that as previously noted it is somewhat questionable whether a lunar shock can develop that is 
similar to the case of the Earth. In the case of the Earth the values of the parameters are such that the radius of the shock surface at the stagnation point is more than 100 times the proton Larmor radius. If one considers the field internal to the magnetosphere the radius of the magnetosphere is some 500 times the proton gyro radius since the magnetic field is 50 gammas or greater at the magnetopause boundary. $6.0 \quad \mathrm{~K}_{\mathrm{P}}$ and Related Studies

During the three month interval, December 1963 to February 1964, solar conditions were reasonably undisturbed. A geomagnetic storm observed on December 2, 1963, at 2117 UT was also measured in interplanetary space by the magnetic field experiment (Ness et al, 1964). During the remainder of the interval however, terrestrial magnetic activity as measured by the planetary index $\mathrm{K}_{\mathrm{p}}$ was generally low. Because the solarterrestrial conditions were relatively quiescent those disturbances which were observed and identified as the lunar wake have not been associated with any solar origin that might cause these variable properties of the interplanetary medium.

Recent studies (Be1l and Defouw, 1964; Davidson and Martyn, 1964; Michel et al, 1964; Stolov and Cameron, 1964) on $\mathrm{K}_{\mathrm{p}}$ have been directed to a resolution of the important question raised by the work of Bigg (1963a, b; 1964) on lunar influences on geomagnetic activity with a synodic period of 29.53 days. The original work was based on the physical supposition that any lunar magnetic field would shield the Earth from propagation of solar particles and thereby affect the level of terrestrial 
magnetic activity. The specific result of this paper is that a turbulent lunar wake associated with a magnetohydrodynamic interaction of the solar wind with the Moon does exist。 If such turbulence in the magnetic field implies equivalent turbulence in the interplanetary plasma it suggests further that fluctuations of magnetic activity could possibly be enhanced at about the time of new Moon.

The statistical significance of the early work has come under severe criticism, although the use of $\mathrm{K}_{\mathrm{p}}$ as a quantity obeying the normal laws of algebra is inherently presumed by all particpants in these discussions. In spite of this, separate but very similar correlative studies by two groups using identical data have been interpreted quite differently. Stolov and Cameron (1964) have concluded that a lunar effect exists but with the interesting result that the phase corresponds to a full Moon disturbance Michel et al. (1964) on the contrary state that their results are not statistically significant. Bell and Defouw (1964) using a superposed epoch method however concluded a lunar effect at full moon does exist statistically.

Davidson and Martyn (1964) using a superposed epoch and harmonic dial analyses on $A_{p}$ conclude that no statistically significant effect exists. These authors conducted "a simple statistical experiment" to illustrate their case. A major problem in the analysis of a dimensionless index such as $\mathrm{K}_{\mathrm{p}}$ or $A_{p}$ is whether or not the statistical significance tests are based on valid statistical properties of the variable being investigated. In the case of these indices it is by no means clear that definitive answers can be obtained. Davidson and 
Martyn (1964) also state that smoothing the data such as done by Bigg can introduce spurious, periodicities. This is not true for the weighting function used by Bigg (running average by three's) and is in general not true for most classical numerical filtering procedures. The general situation with regard to statistical studies on $K_{p}$ or $A_{p}$ is definitely not yet resolved concerning lunar periodicities. Finally a very recent study by Dodson and Hedeman (1964) indicated an anomalous periodicity of 29.5 days in solar cosmic ray data. This latest result is certain to contribute to our apparent lack of understanding of possible lunar related phenomena.

If the geomagnetic tail extends far behind the Earth to the distance of or beyond the Moon, that is at least $60 R_{e}$, then the Moon will interact with the tail and this may lead to temporal variations in the characteristics of the magnetospheric fields and particle populations independent of solar activity. Indeed such an extended magnetospheric tail has recently been proposed by Dessler (1964)。A more pertinent discussion with respect to the magnetic field topology interior to the tail has been given by Axford et al (1964)。Detailed studies on the IMP-1 data obtained while in the geomagnetic tail region are presently being conducted to determine the possible lunar effects during March, April and May of 1964, as well as the detailed topological characteristics of the field. On the basis of these IMP-1 results and others obtained on the Explorer X satellite (Heppner et al (1963)) and Explorer XIV (Cahill (1964) it appears certain that the geomagnetic field and its tail extend far away from the Earth 
in an anti-solar direction. Thus, interaction of the Moon with the Earth's magnetic tail can be reasonably anticipated. The studies of Stolov and Cameron (1964) and Bell and Defouw (1964) lend support to such a conclusion.

Howard et al (1964) have suggested that variations in the electron density in the cislunar medium during a solar eclipse are related to a shadowing effect in the lee of:the'Moon as it interacts with the solar wind. Although the maximum change in solar wind density required to explain the observations is rather high, $100 \mathrm{p} / \mathrm{cm}^{3}$, there is considerable difficulty in accurately removing changes caused by the ionsphere and magnetosphere. This propagation experiment on lunar associated changes in the cislunar medium may indicate a separate technique for the investigation of the magnetohydrodynamic wake of the moon during the time of new moon. 


\section{Summary and Conclusions}

During the period in wh ich the IMP-1 satellite was located in interplanetary space beyond the direct effects of the solar wind interaction with the geomagnetic field a unique disturbance was observed. This was characteristic of the transition region and magnetosphere tail associated with the solar wind interaction with the geomagnetic field. This temporal disturbance showed turbulent magnetic fields and enhanced magnetic flux values well above the normal interplanetary condition. This event is identified as the magnetohydrodynamic wake of the Moon's interaction with the solar wind. The use of an aerodynamic analogy, which has proven so successful in studying the Earth's interaction with the solar wind, shows that the satellite was within the primary shock region associated with a super Alfvénic solar wind flow. A second opportunity to investigate the lunar wake in January 1964 was obscured by the location of the satellite within the shock region of the solar wind interaction with the geomagnetic field. A third opportunity in February 1964, possibly detected a wake but it did not demonstrate the same characteristic features. At this time the satellite was some 200 lunar radii from the Moon.

The great distance at which the lunar wake is observed and the lack of quantitative descriptions regarding the Earth's magnetic tail preclude an estimate of the strength of any lunar magnetic field. However, the fact that a lunar wake was observed during December 1963 is suggestive of a lunar magnetic 
field at the equatorial surface of several hundred gammas or more .

A major question raised by this paper is whether or not a shock wave due to the moon is expected to develop at all times in the interplanetary medium unless an intrinsic lunar magnetic field is present. It would appear that the absence of a strong field, and the small size of the Moon preclude a condition analogous to that in the case of the Earth in which a shock wave is usually expected to occur. The Larmor radius of the average solar wind proton is of the same order of magnitude as the lunar radius and thus the analogy with fluid dynamics is probably not justified.

The Russian measurements on the Lunik II spacecraft impacting the lunar surface indicate that the magnetic field of the Moon is not sufficently strong to produce field strengths larger than 100 gammas at a distance of $55 \mathrm{Km}$. This can be understood on the basis of a compression of a lunar magnetic field by the solar wind. However, at the same time that this data was obtained scintillation counter data (Vernon et al, 1960) obtained at a distance of less than $1000 \mathrm{Km}$ from the lunar surface indicated energetic particle flux values 100 times those found typically in interplanetary space. This value of $1000 \mathrm{Km}$ is tantalizingly close to the lunar shock wave stand off distance. Indeed the magnetic field data for distances less than $1000 \mathrm{~km}$ are also larger and more variable also. This may indicate that shock region characteristics similar to that observed surrounding 
the Earth may have been present surrounding the Moon at that time.

The fact that the Earth's magnetospheric tail does extend far behind the Earth may well lead to lunar associated variations of $K_{p}$ or $A_{p}$ at full moon as the Moon sweeps through the Earth's tail. 
Acknowledgements

I am indebted to C. S. Scearce and J. B. Seek for their important participation in the IMP-1 magnetic field experiment. Stimulating discussions of the lunar wake with Drs. E.K. Bigg, A. G。W. Cameron and T. Obayashi are hereby acknowledged. The comments of Dr.J. M. Wilcox are particularly appreciated. 
Anderson, K. A., H. K. Harris and R. J. Paoli, Energetic Electron fluxes in and beyond the Earth's Outer Magnetosphere, preprint, 1964 .

Axford, W. I., The interaction between the solar wind and the Earth's magnetosphere, J. Geophys. Res., 67, 37913796,1962 .

Axford, W. I., H. E. Petschek and G. L. Siscoe, The Tail

of the Magnetosphere, AVCO-Everett Research Report R190, August, 1964

Beard, D. B., The interaction of the terrestrial magnetic field with the solar corpuscular radiation, J. Geophys. Res.,65, 3559-3568, 1960 。

Be1l, B. and R. J. Defouw, Concerning a Lunar Modulation of Geomagnetic Activity, J. Geophys. Res., 69, 3169-3174, 1964. Bernstein, W., R. W. Fredricks, J.L. Vogl and William A. Fowler, The Lunar Atmosphere and the Solar wind, Icarus $2,233-248,1963$.

Biermann, L., Kometenschweife and Solare Korpuscular Strahlung, Z Astrophys. $29,274-286,1951$.

Bigg, E. K., The influence of the Moon on geomagnetic disturbances, J. Geophys. Res. 68 1409-1413, 1963a.

Bigg, E. K。, Lunar and Planetary Influences on Geomagnetic Disturbances, J. Geophys. Res., 68 099-4104, 1963b. 
Bigg, E. K., Lunar Influences on the Frequency of Magnetic Storms, preprint , 1964.

Bonetti, A., H. S。Bridge, A. J. Lazarus, B。Rossi and F。 Scherb, Explorer 10 plasma measurements, J.Geophys. Res., 68, 4017-4063, 1963 .

Bridge, H., A. Egidi, A. Lazarus, E. Lyon and L。 Jacobson Preliminary results of plasma measurements on IMP-A, COSPAR presentation, Florence, Italy, 1964 。

Cahill, L。J。and R. G. Amazeen, The Boundary of the Geomagnetic Field, J。Geophys. Res., 68, 1835-1844, 1963 .

Cahill, L. J., Preliminary Results of Magnetic Field Measurements in the tail of the Geomagnetic cavity, IGY Bulletin 79,1964

Chapman, S。, Solar plasma, geomagnetism and aurora in geophysics, in The Earth's Environment, Les Houches 1962 Lectures, edited by C. Dewitt et al, pp. 371-502, Gordon and Breach, New York, 1963.

Chapman, S. and J. Bartels, Geomagnetism, Clarendon Press, Oxford, 1940 .

Davidson, T.W. and D. F. Martyn, A Supposed Dependence of Geomagnetic Storminess on Lunar Phase, J. Geophys. Res., $63,3973-3979,1964$ 。

Dassler, A. J., Length of the Magnetospheric Tail, J。Geophys. Res., 69, 3913-3918, 1964 。

Dodson, Helen W. and E. Ruth Hedeman, An unexpected effect in Solar Cosmic Ray Data related to 29.5 Days, J.Geophys. Res., $69,3965-3971,1964$ 。 
Dolginov, S. H., E. G. Eroshenko, L。N。Zhugov, N. V. Pushkov and L. O. Tynrmina, Investigation of the Magnetic Field of the Moon, Geomagnetism and Aeronomy, 1, 21-29, 1961.

Doodson, A. T., Oceanic tides, Advan. in Geophys., 5, 118-152, Academic Press, New York, 1958 .

Elsasser, W。M., The Earth's Interior and Geomagnetisli, Revs. Mod。Phys., 22, 1-35, 1950 .

Fejer, J. A., Atmospheric tides and associated magnetic effects, Rev. of Geophysics, 2,275-310, 1964。

Freeman, J。W。, J. A。, Van Allen and L. J。, Cahill, Explorer 12 observations of the magnetosphere boundary and the associated solar plasma on Sept。13, 1961, J。Geophys. Res., 68, 2121-2130, 1963 .

Freeman, J. Wo, Electron distribution in the outer radiation zone, J. Geophys。Res。, 69, 1691-1724, 1964 。

Gold, T。, The magnetosphere of the Moon, Cornell Univ。, Report CTSR-177, 1964。

Gringauz, K。 I。, Some results of experiments in interplanetary space by means of charged particle traps on Soviet space probes, Space Research II, 539-553, North-Holland, Amsterdam, 1961 .

Hapke, B. W。, Effect of the Solar Wind on the photometric properties of the lunar surface, Trans. A. G. U., 45,347, 1964 。 
Harrison, E. R., Solar Corpuscular radiation and the nature of the Earth's Immediate Interplanetary environment, Proc。 Int. School of Physics "Enrico Fermi", Course XIX, 65-84, edited by B. Peters, Academic Press, New York, 1963.

Hayes, W. D。 and R. F。 Probstein, Hypersonic Flow theory", Academic Press, New York, 1959.

Heppner, J。P。, N. F。Ness, T。L。Skillman and C。S.Scearce, Explorer X Magnetic Field Measurements, J。Geophys. Res., $68,1-46,1963$.

Hida, K., An approximate study on the detached shock wave in front of a circular cylinder and a sphere, J. Phys. Soc. Japan, 8, 740-745, 1953 .

Hinton, F。 L。 and D. R. Taeusch, Variation of the lunar atmosphere with the strength of the solar wind, J.Geophys。 Res., 69, 1341-1347, 1964 .

Howard, H。 T。, B. B。 Lusignan, P. Yoh and V. R。 Eshleman, Radar Doppler and Faraday Polarization Measurements of the Cislunar Medium during the July 20, 1963 Solar Eclipse, J . Geophys. Res, 69, 540-544, 1964 。

Imai, I., On Flows of Conducting Fluids past Bodies, Revs。 Mod. Phys., $32,992-999,1960$.

Inglis, D. R。, Theories of the Earth's Magnetism, Revs. Mod. Phys。, 27,212-248, 1955 。

Johnson, F。S., The gross character of the geomagnetic field in the solar wind, J. Geophys. Res., 65, 3049-3051, 1960. 
Kantrowitz, A. and H. E. Petschek, MHD Characteristics and Shock Waves, AVCO-Everett Research Report Rl85, July, 1964 .

Kellogg, P.J., Flow of plasma around the earth, J. Geophys. Res., 67, 3805-3811, 1962 .

Kopal, Z. and T. W. Rackham, Excitation of Lunar luminescence by Solar flares, Nature, 201 (4916), 239-241, 1964。

Lees, L., Interaction between the solar plasma wind and the geomagnetic cavity, AIAA preprint 64-88, New York, 1964 Lees, L。 and L. Hromas, Turbulent diffusion in the wake of a blunt nosed body at hypersonic speeds, J. Aero. Sci。, $29,976-993,1962$.

Levin, B., Thermal History of the Moon, in The Moon edited by Kopal and Mikhailov, pp. 157-167, Academic Press, New York, 1960 .

Levy, R。H。, H。E。Petschek and G. L. Siscoe, Aerodynanic Aspects of the Magnetosphere Flow, AVCO-Everett-RI70, 1964 .

Ludford, G。S。S。 and J。D. Murray, on the flow of a conducting fluid past a magnetized sphere, J. F1. Mech., 7, 516-528, 1960 。

MacDonald, G. J. F., Interior of the Moon, Science, 133,1045-1050, 1961.

MacDonald, G. J。F。, Internal constitution of the inner planets and the Moon, Space Science Review, 2, 473-557, 1963 .

Marochnik, L. S., The Interaction of Solar Corpuscular Streams with the Atmospheres of Comets, Geomagnetism and Aeronomy, $3,608-615,1963$. 
Melchior, Paul, Earth Tides, Advan。in Geophys. 4, 392-445,

Academic Press, New York, 1957.

Michel, F. G. A. J。Dessler and G. Ko Walters, A Search for

Correlation Between $\mathrm{K}_{\mathrm{p}}$ and the Lunar Phase, J. Geophys. Res。, 69, 4177-4181, 1964 .

Michel, F。G., Detectability of disturbances in the Solar

Wind, J. Geophys. Res., to appear, 1964 .

Ness, N。F., C.S。 Scearce, J。B。 Seek, Initial results of

the IMP-1 Magnetic Field Experiment, J. Geophys。Res.,

$69,3531-3570,1964$ 。

Ness, N. F。 and J。M.Wilcox, The solar origin of the Inter-

planetary Magnetic Field, Phys。Rev。Letters, 13 (15), 461-4, 1964.

Neugebauer, M., Question of the existence of a lunar magnetic

field, Phys。Rev。Letters, 4 (1), 6-8, 1960 。

Obayashi, T., Interaction of Solar plasma Streams with the outer

Geomagnetic field, J. Geophys。Res., 69, 861-868, 1964.

Parker, E。N., Dynamics of the interplanetary gas and magnetic

field, Astrophys。J。, 128, 664-676, 1958 .

Parker, E。 No, The hydrodynamic theory of solar corpuscular

radiation and stellar winds, Astrophys.J., 132, 821-866, 1960 .

Parker, E. N。, Interplanetary Dynamical processes, pp。131, Interscience, New York 1963 .

Piddington, J。H., Geomagnetic storm theory, J. Geophys, Res., $65,93-105,1960$.

Sears, W. R。, Some remarks about flow past bodies, Revs。 Mod. Phys., 32, 701-705, 1960 。 
Siebert, M。, Atmosphere tides, Advan。in Geophys。 7, 105-187, Academic Press, New York, 1960.

Snyder, C. and Neugebauer, Interplanetary solar wind measurements by Mariner 2, Space Research IV, edited by P. Muller, North-Holland Publ. Co., Amsterdam, 1964.

Spreiter, J.R. and W. P。 Jones, On the effect of a weak interplanetary magnetic field on the interaction between the solar wind and the geomagnetic field, J. Geophys. Res., $68,3555-3565,1963$ 。

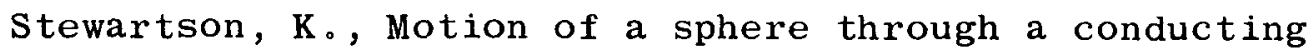
fluid in the presence of a strong magnetic field, Proc.Camb。Phil.Soc., 52,301-316, 1956.

Stolov, H. L. and A. G。W. Cameron, Variations of Geomagnetic Activity with Lunar Phase, J. Geophys. Res., to appear, 1964. Vernov, S。N。, A. E. Chudakov, P。V.Valukov, Yu。I。Logachev and A.G. Nikolayev, Radiation measurements during the flight of the second Moon Rocket, Dokl。Acad. Nauk SSSR, $130,517-520,1960$.

Vestine, E.H., Utilization of a Moon-Rocket System for Measurement of the Lunar Magnetic Field, RAND report RM-1933，1957。

Wehner, G。K., C。E. Kenknight and D。 Rosenberg, Modification of the lunar surface by the solar-wind bombardment, planet. Space Sci。, 11,1257-1261, 1963 。 
Zharkov, V. N. and F. R. Ulinich, Can the Moon sustain a magnetic field by hydromagnetic amplification?, Proceedings of Geophysical Institute of O. Yn. Shmidt No. 11 (178), 50-55, 1960. (Translated for NSF 1963). 


\section{LIST OF FIGURES}

1. Lunas magnetic cavity radius of curvature $\left(R_{c}\right)$ in units of lunar radius $\left(R_{m}\right)$ as a function of the solar wind flux and equatorial field strength $\left(B_{0}\right)$ of assumed lunar dipole.

2. Relative positions of IMP-1 satellite, earth and moon projected on ecliptic plane during period December 12-16, 1963 when the wake of the moon was detected in interplanetary space.

3. Relative positions of IMP-1 satellite, earth and moon projected on solar ecliptic $\mathrm{Y}-\mathrm{Z}$ plane during period December $12-16,1963$ when the wake of the moon was detected in interplanetary space.

4. Variation of relevant earth, moon and IMP-1 satellite position parameters during period December 12-16, 1963. The wake of the moon was detected between 0130 December 14 to 0900 December 15. The time intervals of turbulence and of unusually strong fields are indicated.

5. Magnetic field data from orbit No. 5 December 14, 1963 illustrating IMP-1 entry into lunar magnetohydrodynamic wake from relatively unaisturbed interplanetary space at 0130 .

6. Magnetic field data from orbit No. 5 December 15, 1963 illustrating IMP-1 exit from lunar magnetohydrodynamic wake into relatively undisturbed interplanetary space at 0900 with large field magnitudes observed bwtween 1800 to 2100 December 14 .

7. Relative positions of IMP-1 satellite, earth and moon projected on ecliptic plane during period January 11-15, 1964 . 
8. Relative positions of IMP-1 satellite, earth and moon projected on solar ecliptic $\mathrm{Y}-\mathrm{Z}$ plane during period January $12-16,1964$.

9. Variation of relevant earth, moon and IMP-1 satellite position parameters during period January 12-16, 1964.

10. Relative pusitions of IMP-1 satellite, earth and moon projected on ecliptic plane during period February 9-14, 1964 when a weak wake of the moon was possibly observed.

11. Relative positions of IMP-1 satellite, earth and moon projected on solar ecliptic $\mathrm{Y}-\mathrm{Z}$ plane during period February 9-14, 1964 when a weak wake of the moon was possibly observed.

12. Variation of relevant earth, moon and IMP-1 satellite position parameters during period February 10-15 when a weak wake of the moon was possibly observed between 0400 to 1200 February 10 .

13. Magnetic field data from orbit No. 20, February 10, 1964 , illustrating IMP-1 sampling of the interplanetary magnetic field and possible lunar magnetohydrodynamic wake after exit from transition region of the solar wind interaction with the geomagnetic field. The Earth's shock wave was traversed at a geocentric distance of $30.3 \mathrm{R}_{\mathrm{e}}$.

14. Magnetic field data from orbit No. 20, February 11, 1964, illustrating IMP-1 sampling of the interplanetary nagnetic field and possible lunar magnetohydrodynamic wake before entry into transition region of solar wind interaction with geomagnetic field. The earth's shock was traversed at a 
geocentric distance of $23.6 \mathrm{R}_{\mathrm{e}}$ with a possible "precursor" detected at $24.2 \mathrm{R}_{\mathrm{e}}$.

15. The Alfven velocity as a function of the interplanetary magnetic field strength and solar plasma flux parameters. The average magnetic field in interplanetary space was 5 gammas with 1 imits of 1 to $10 \gamma$.

16. Mach angle as function of the corresponding Alfvén mach number. 17. Relative position of IMP-1 satellite and moon in lunar associated coordinates illustrating envelopment within lunar shock wave assuming aerodynamic similitude during December 1963 and February 1964. 


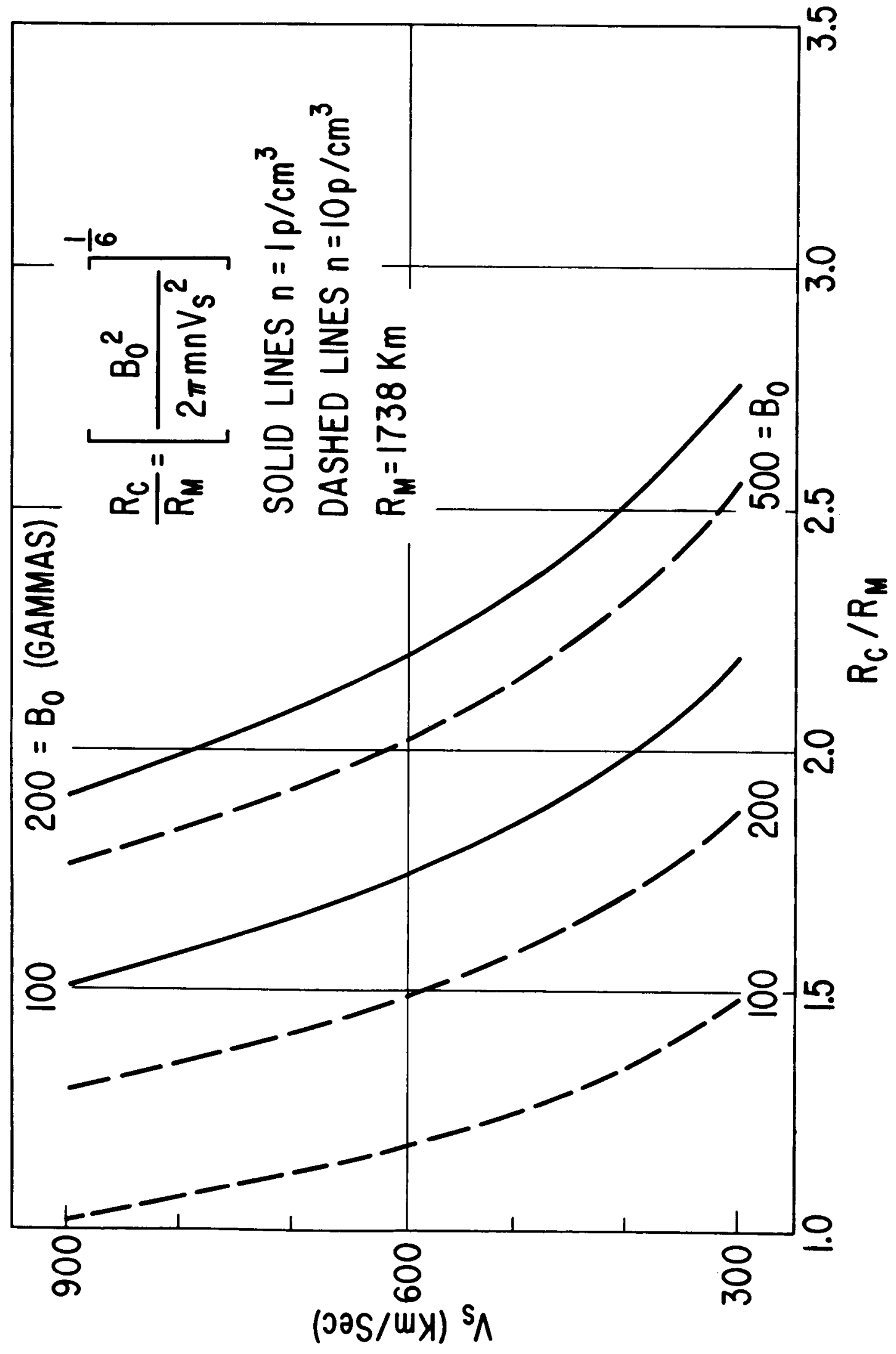




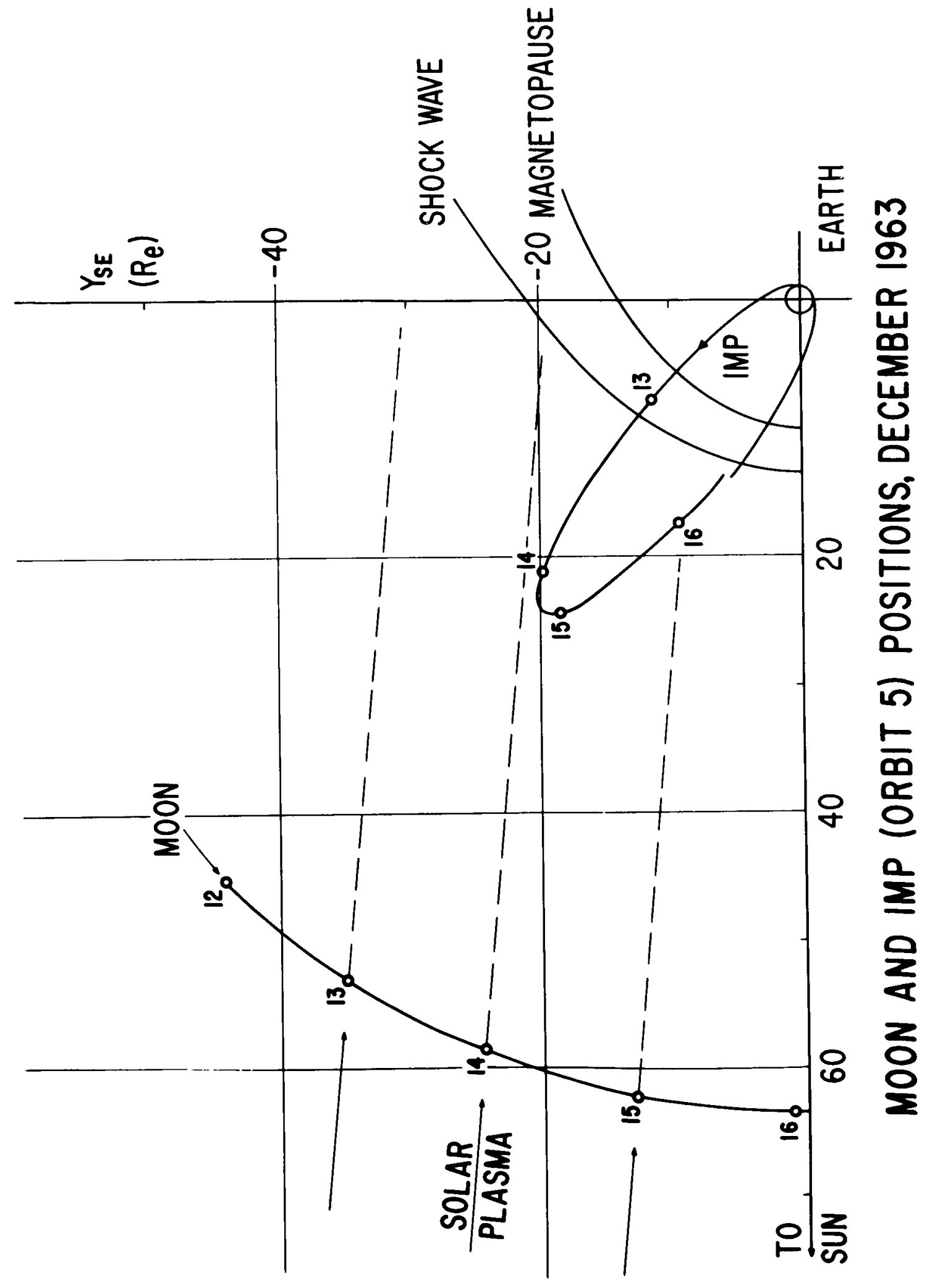




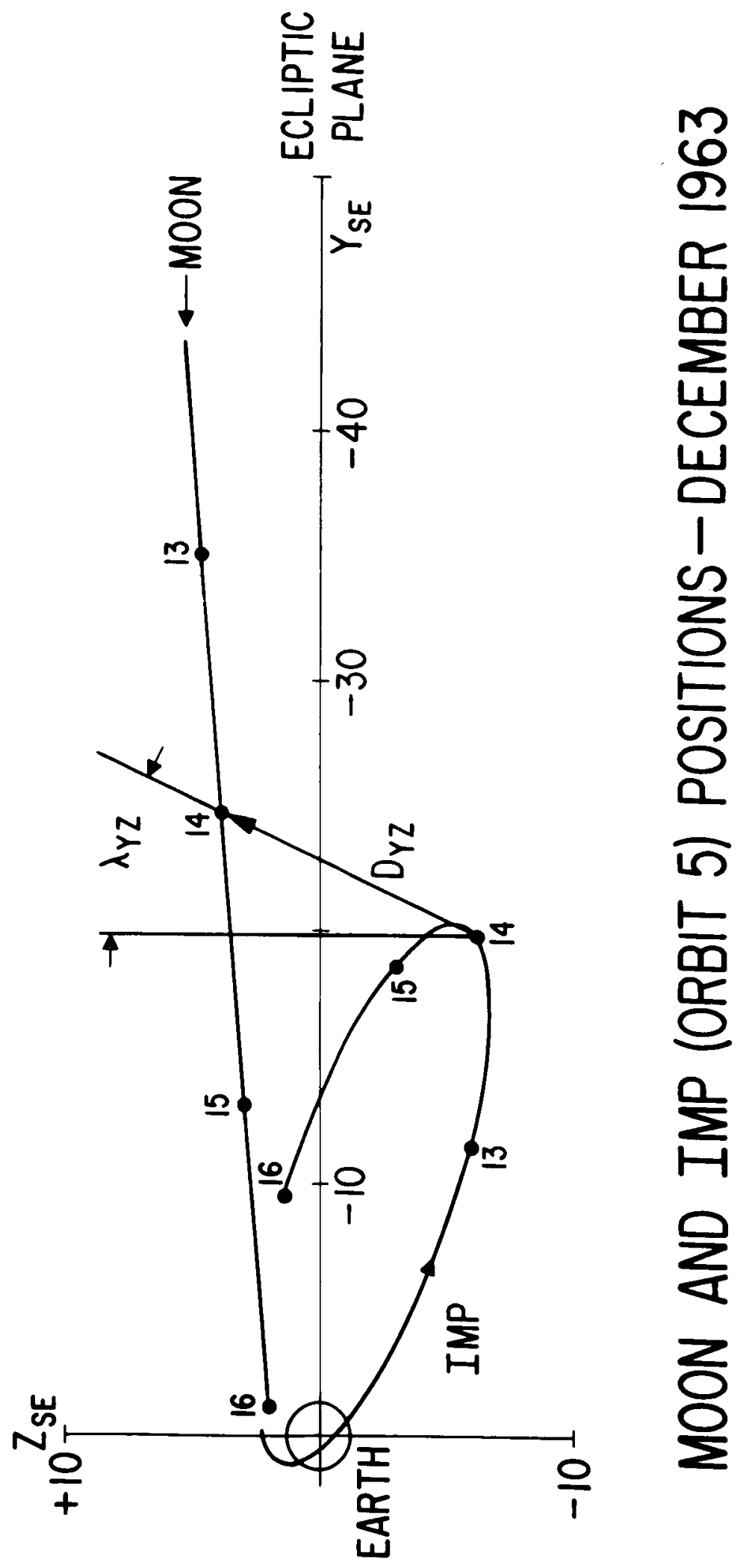




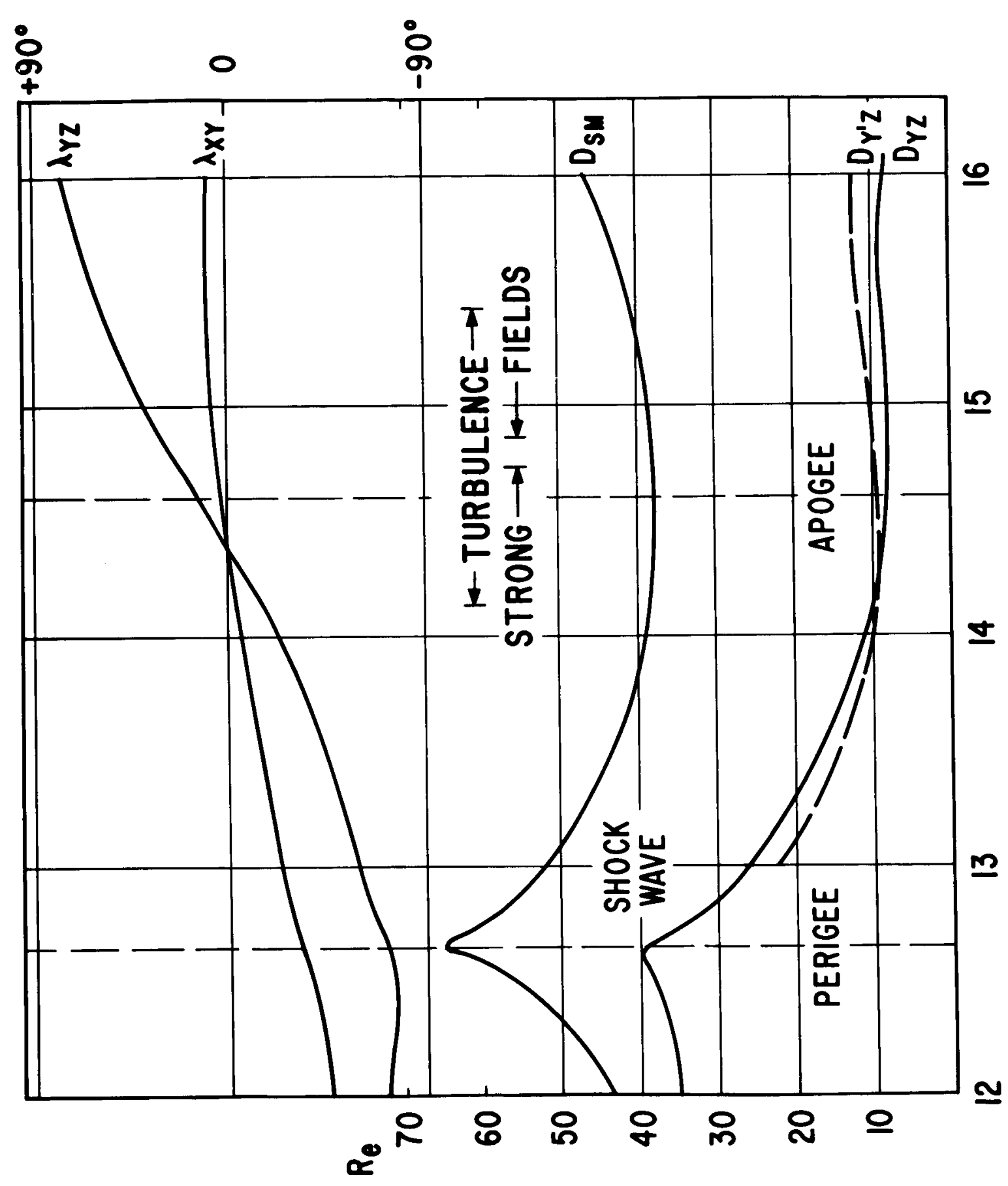

$m$

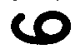

o

告

$\frac{2}{2}$

$\frac{2}{2}$

$\sum$

닌

a 


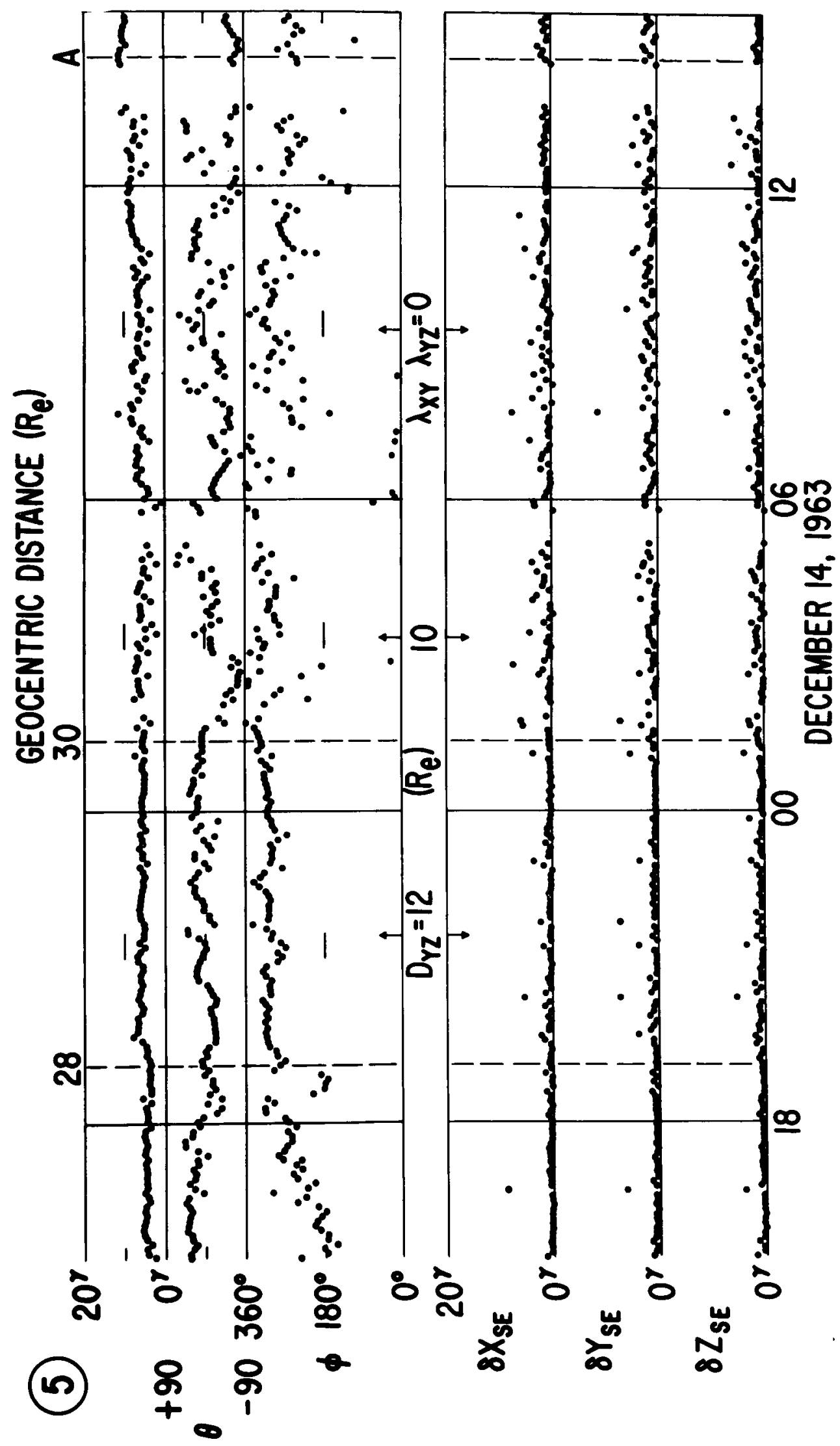




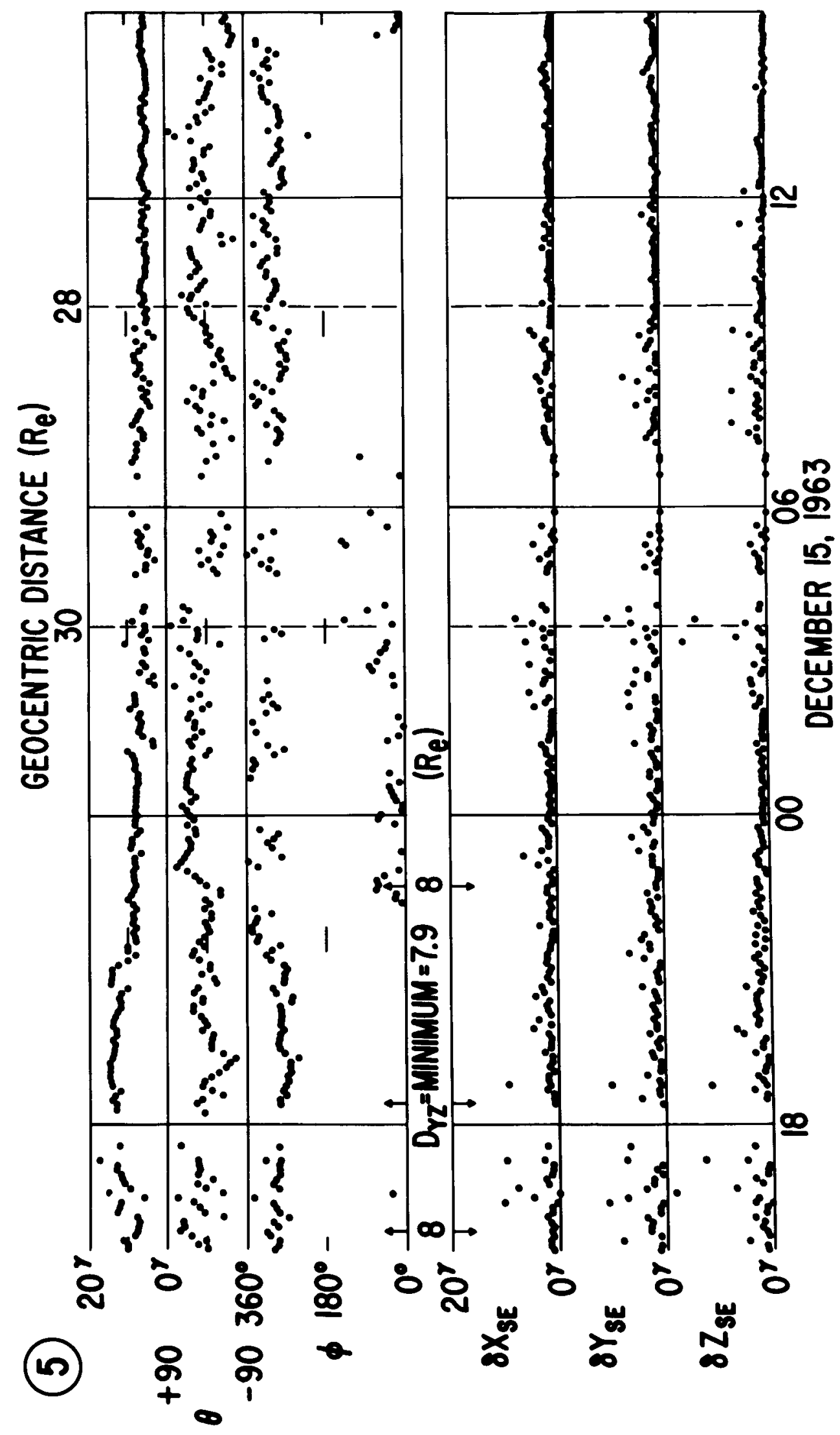




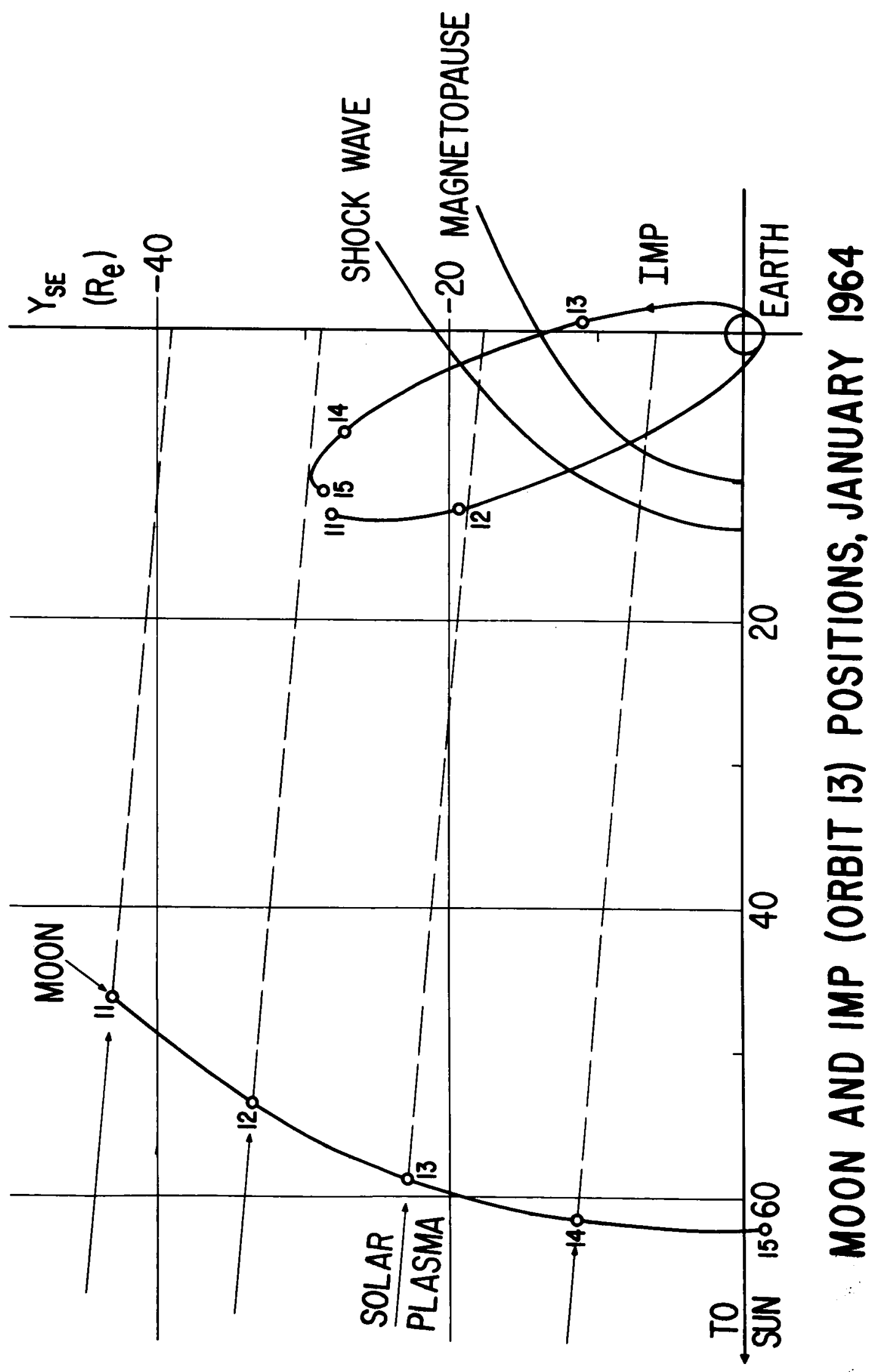




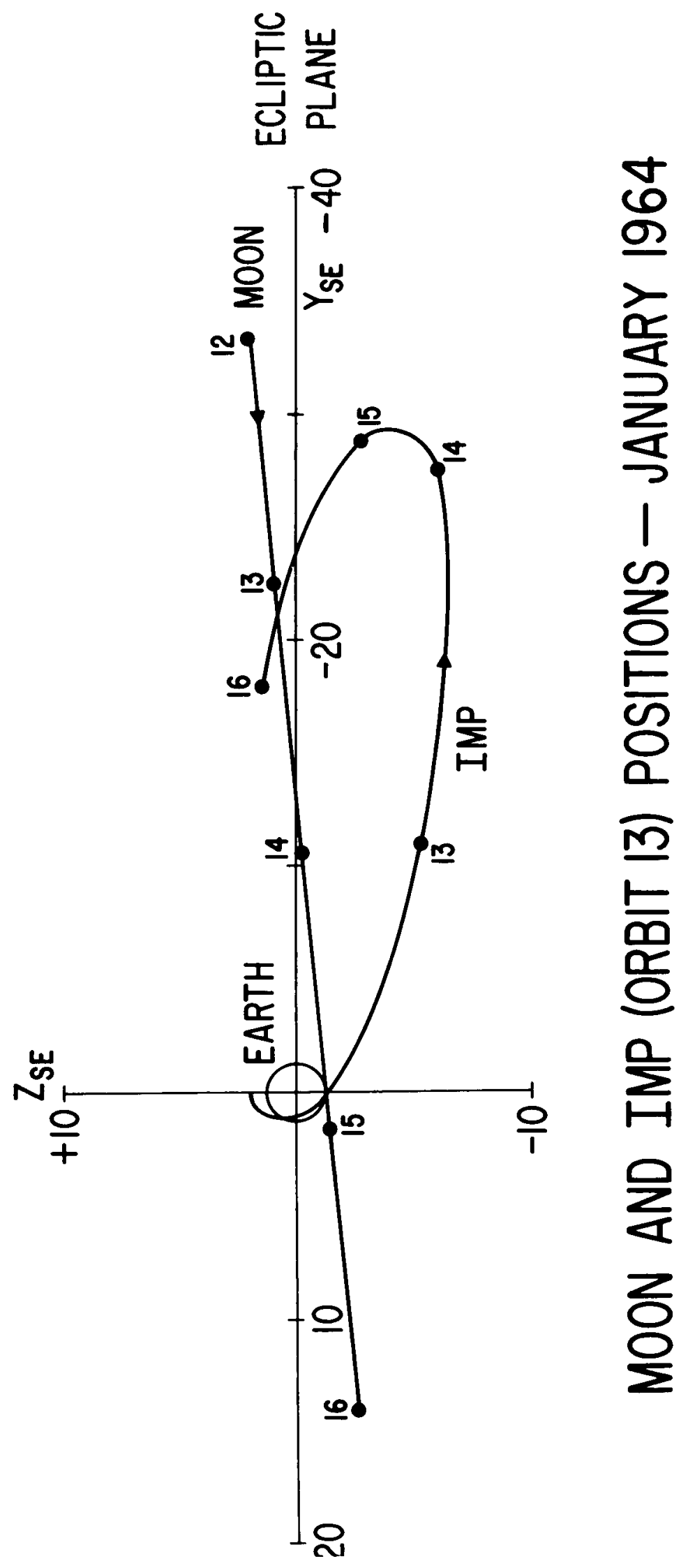




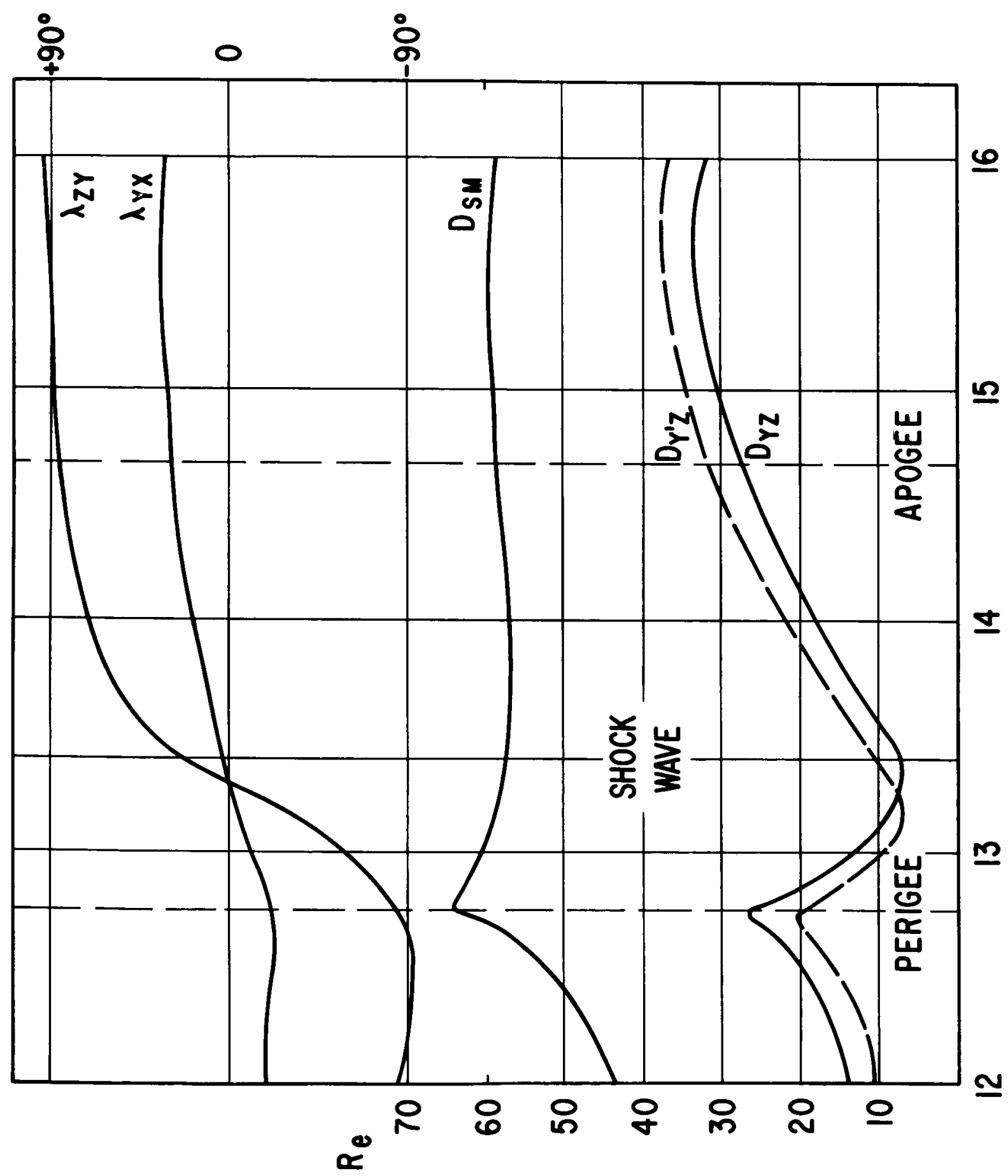

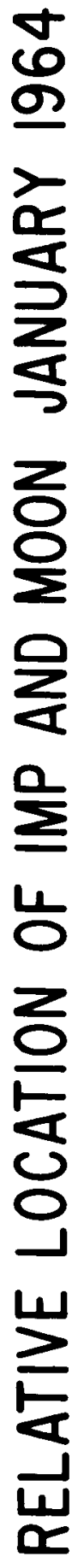




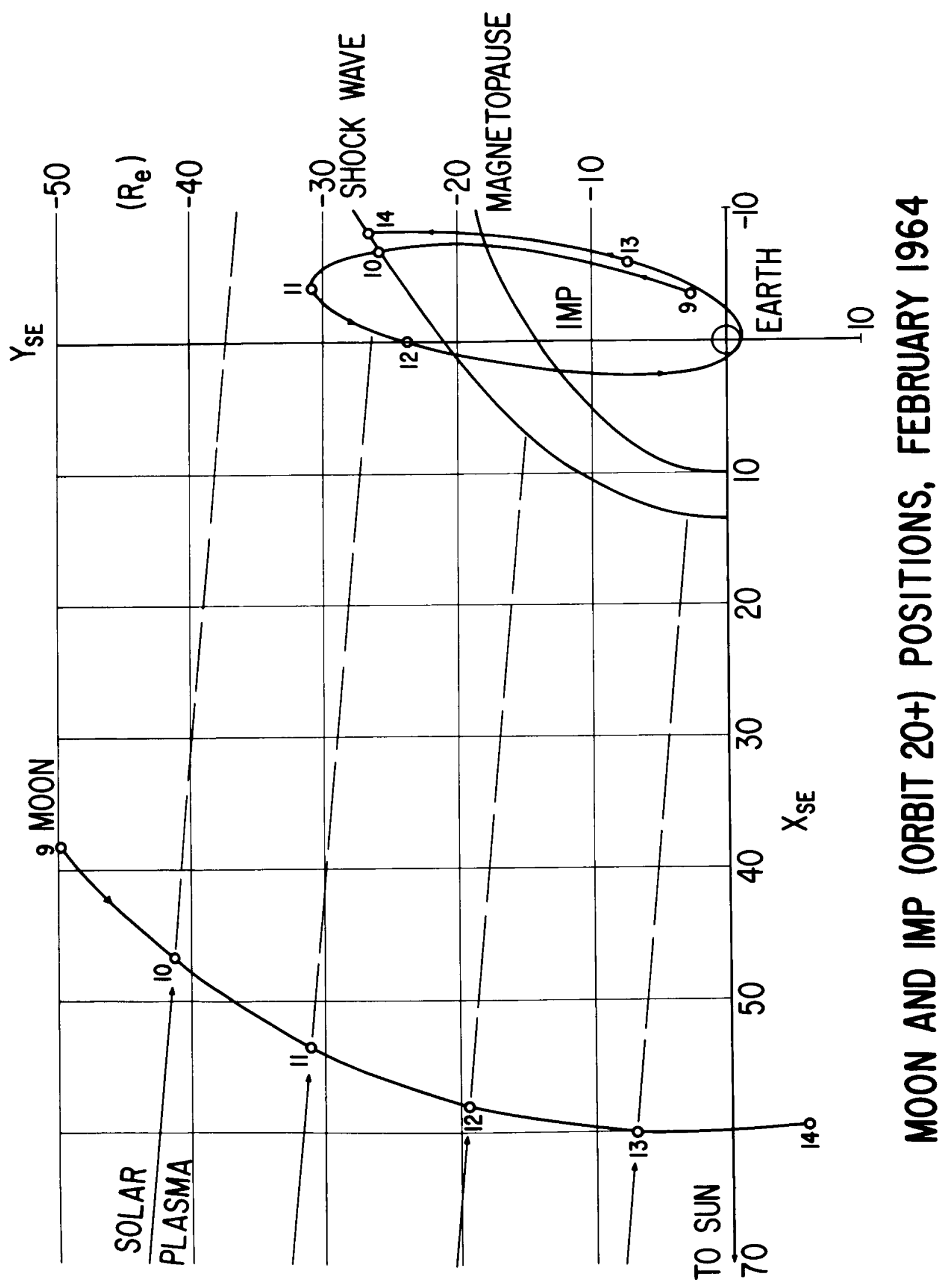




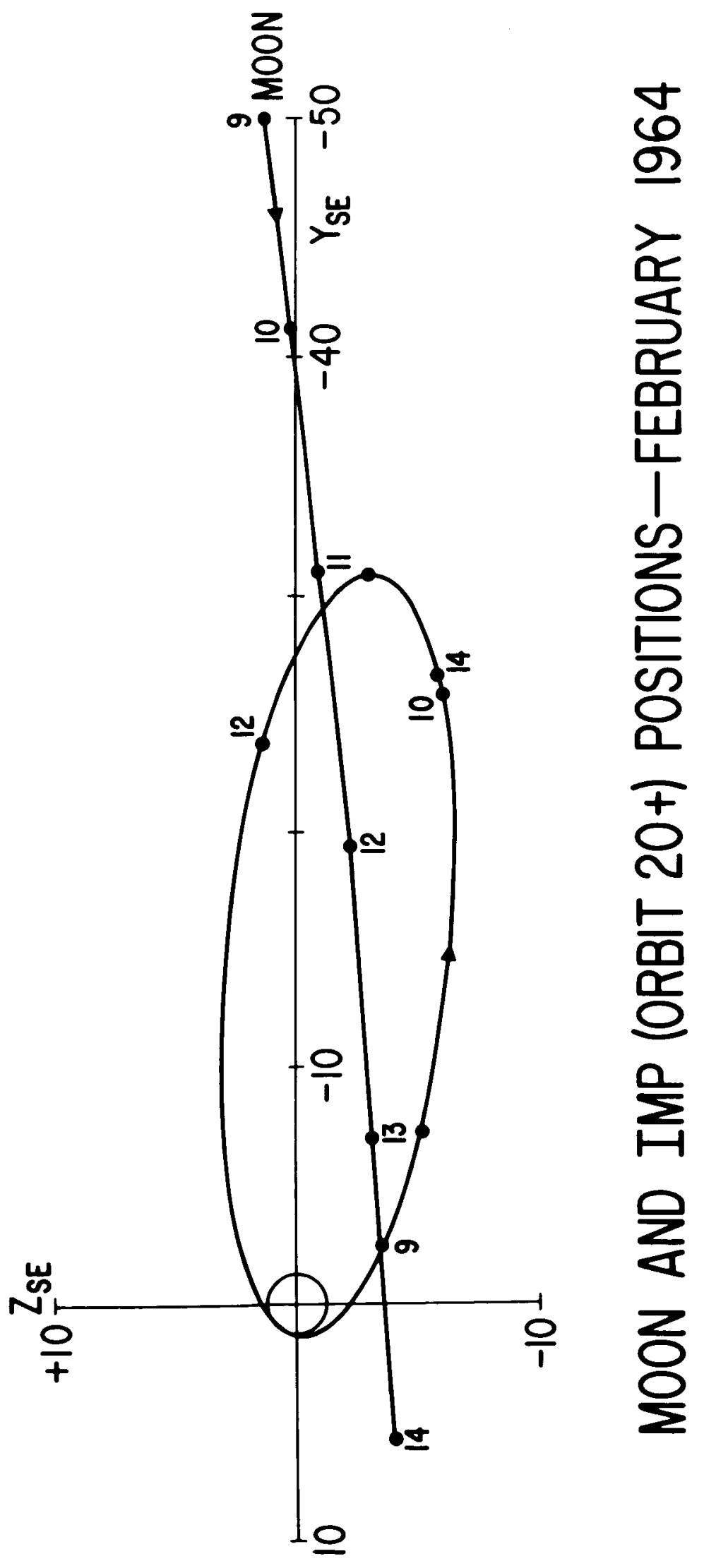




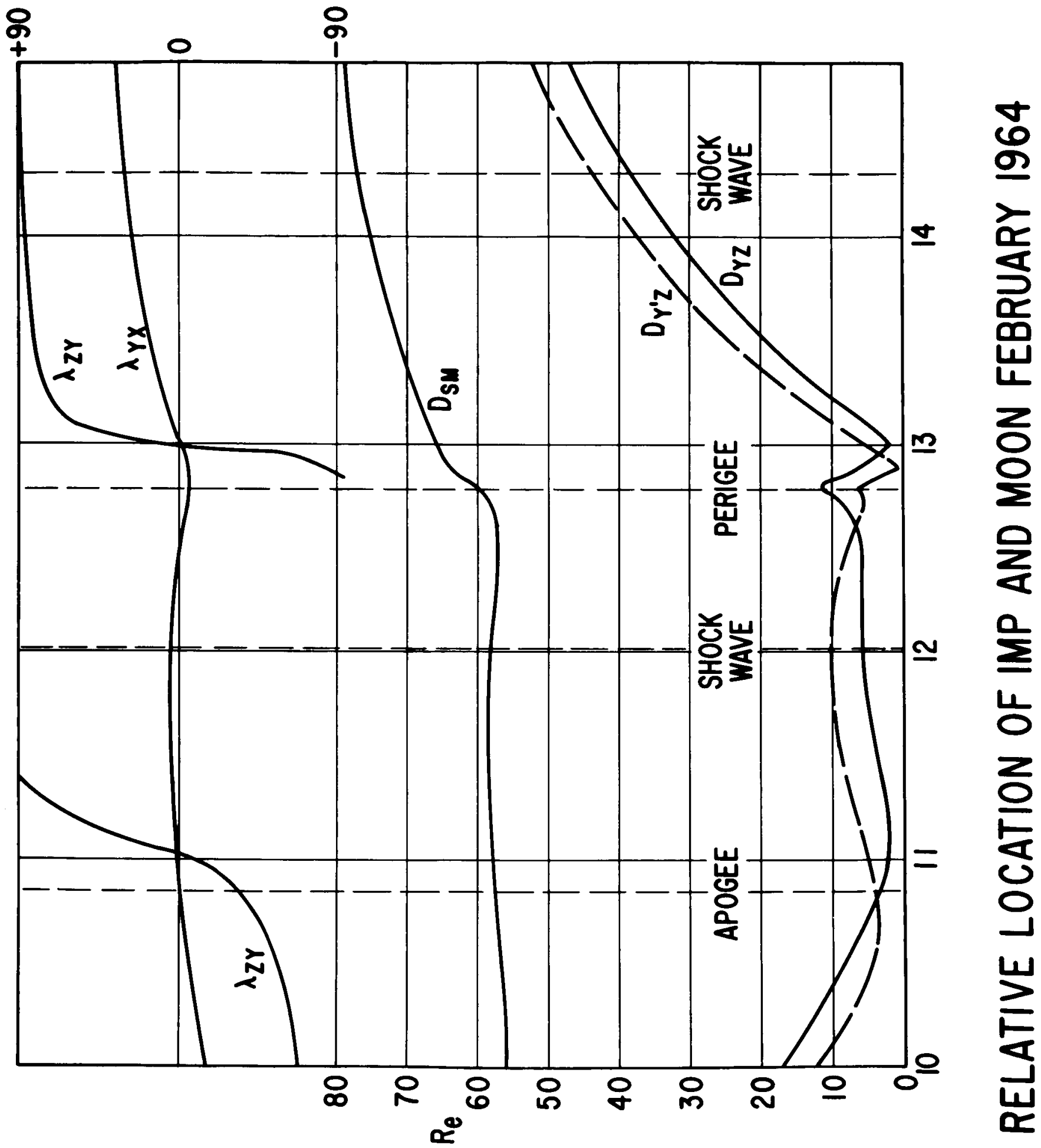




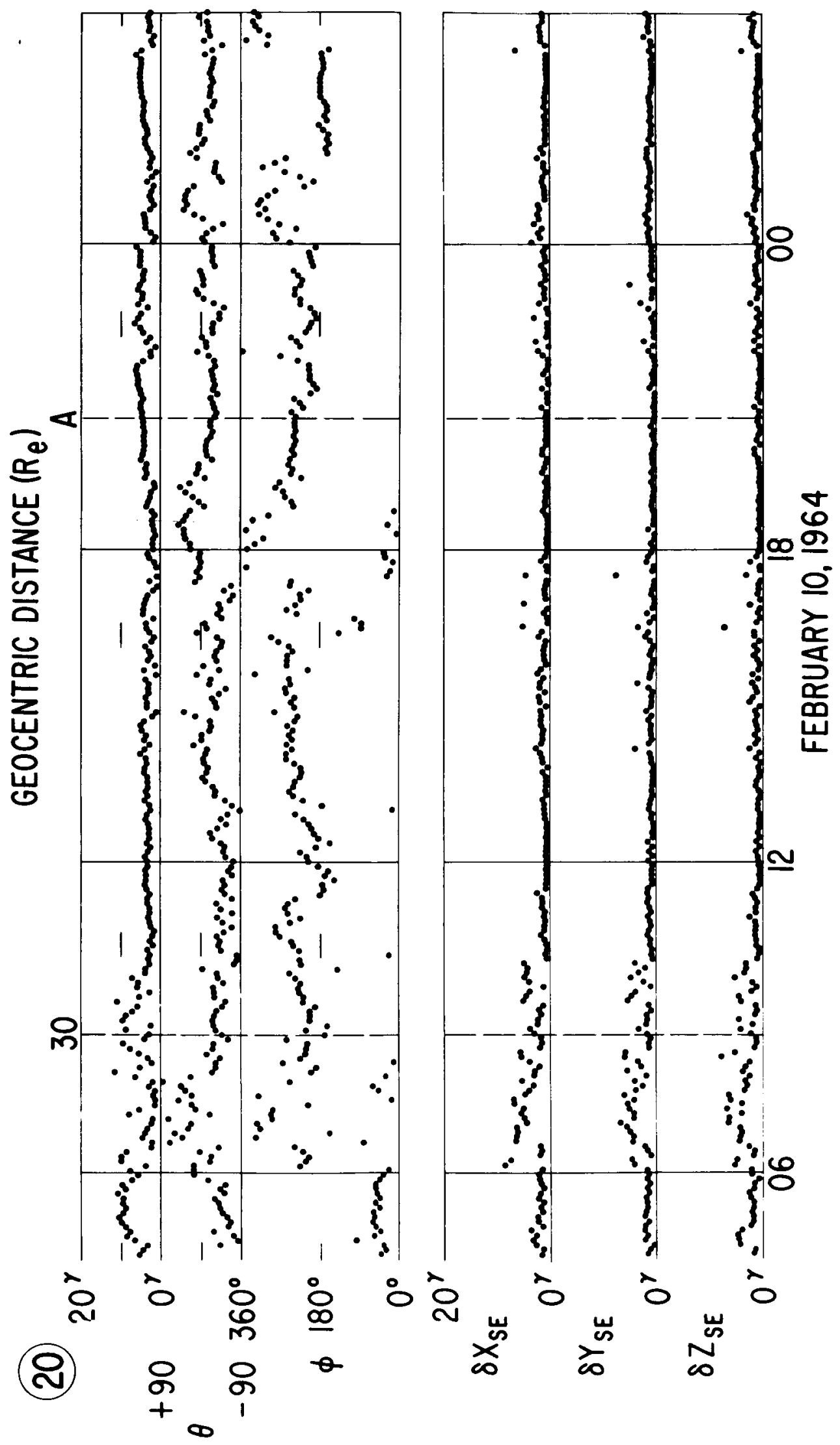




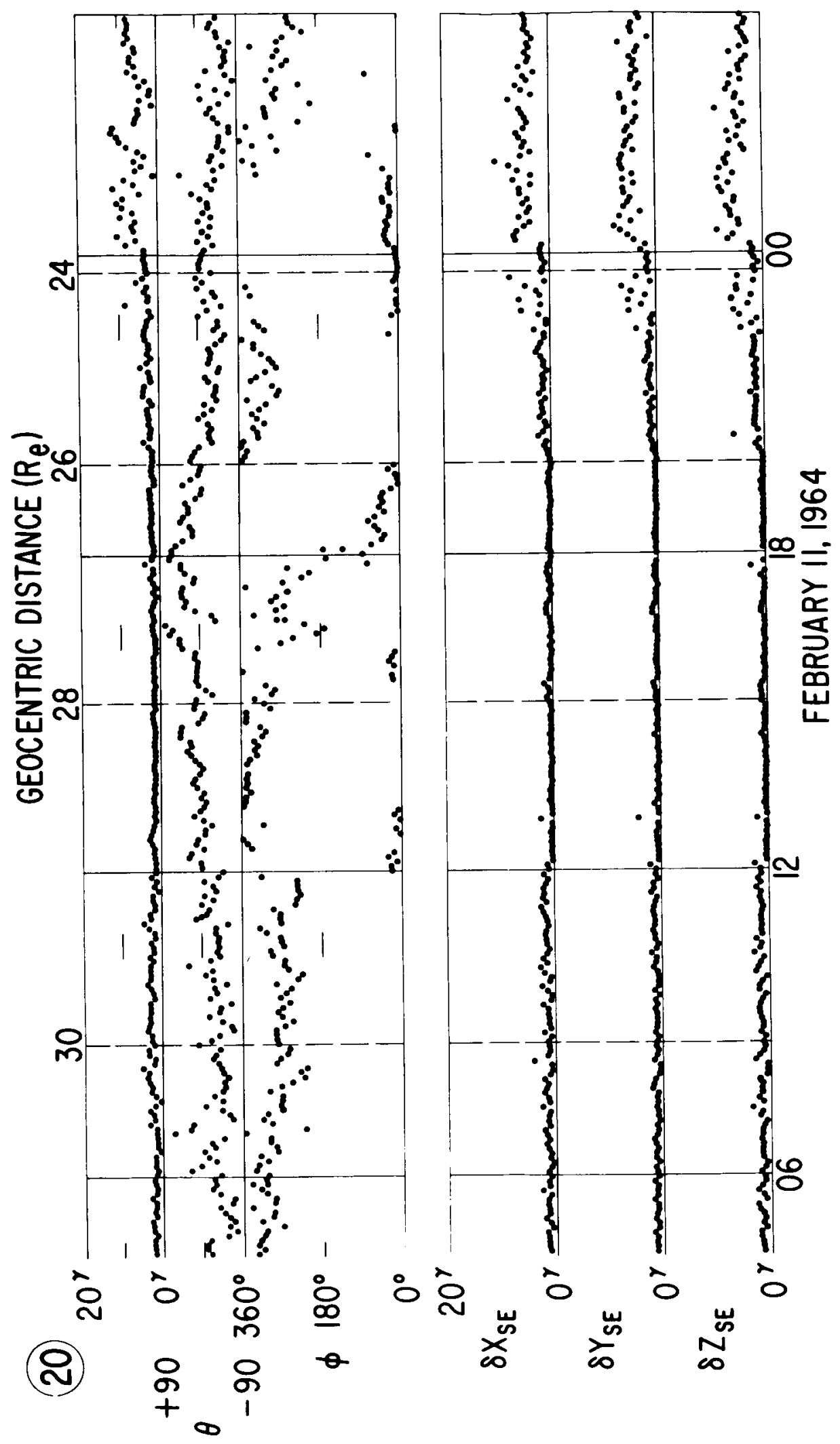




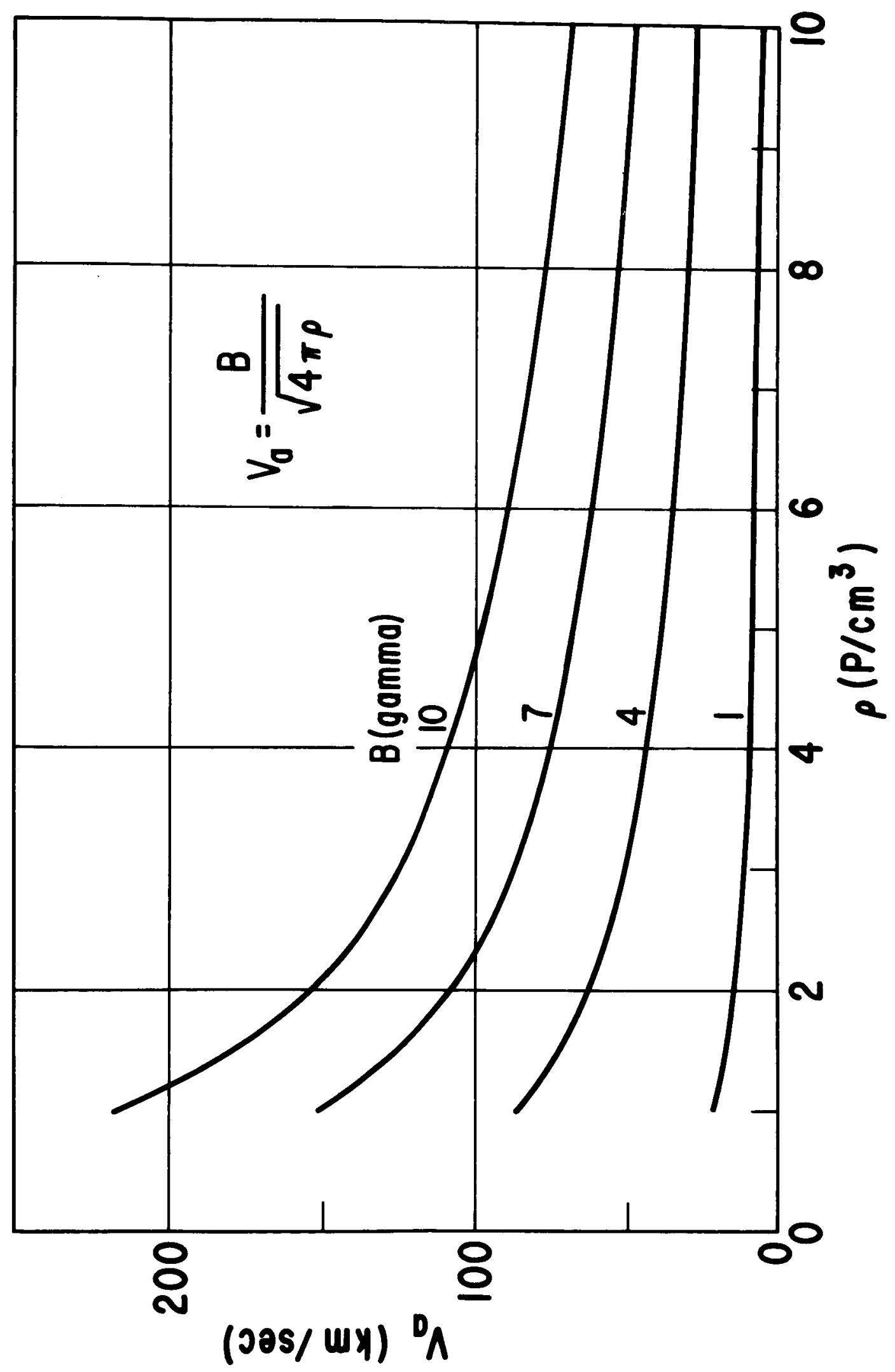




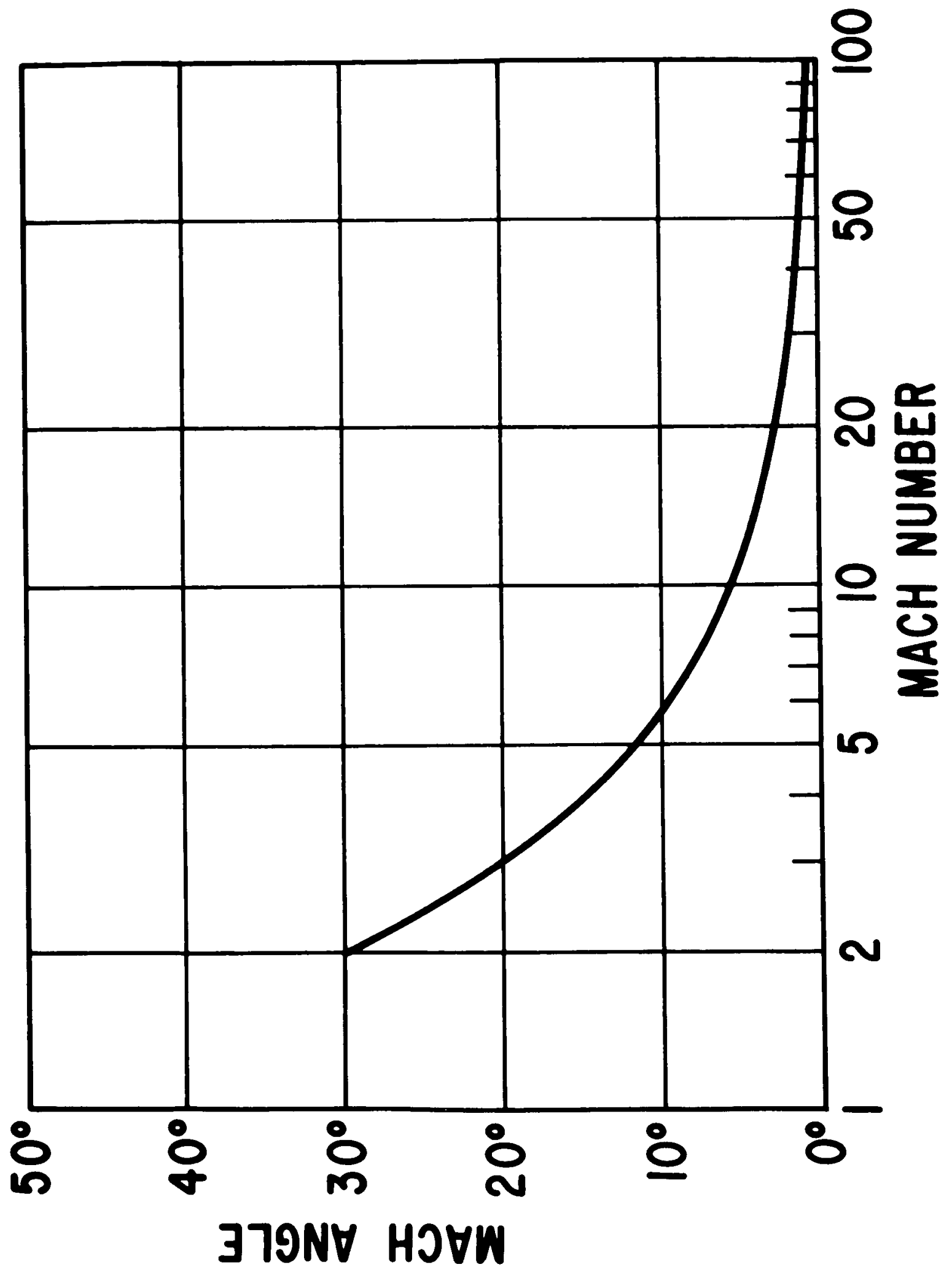




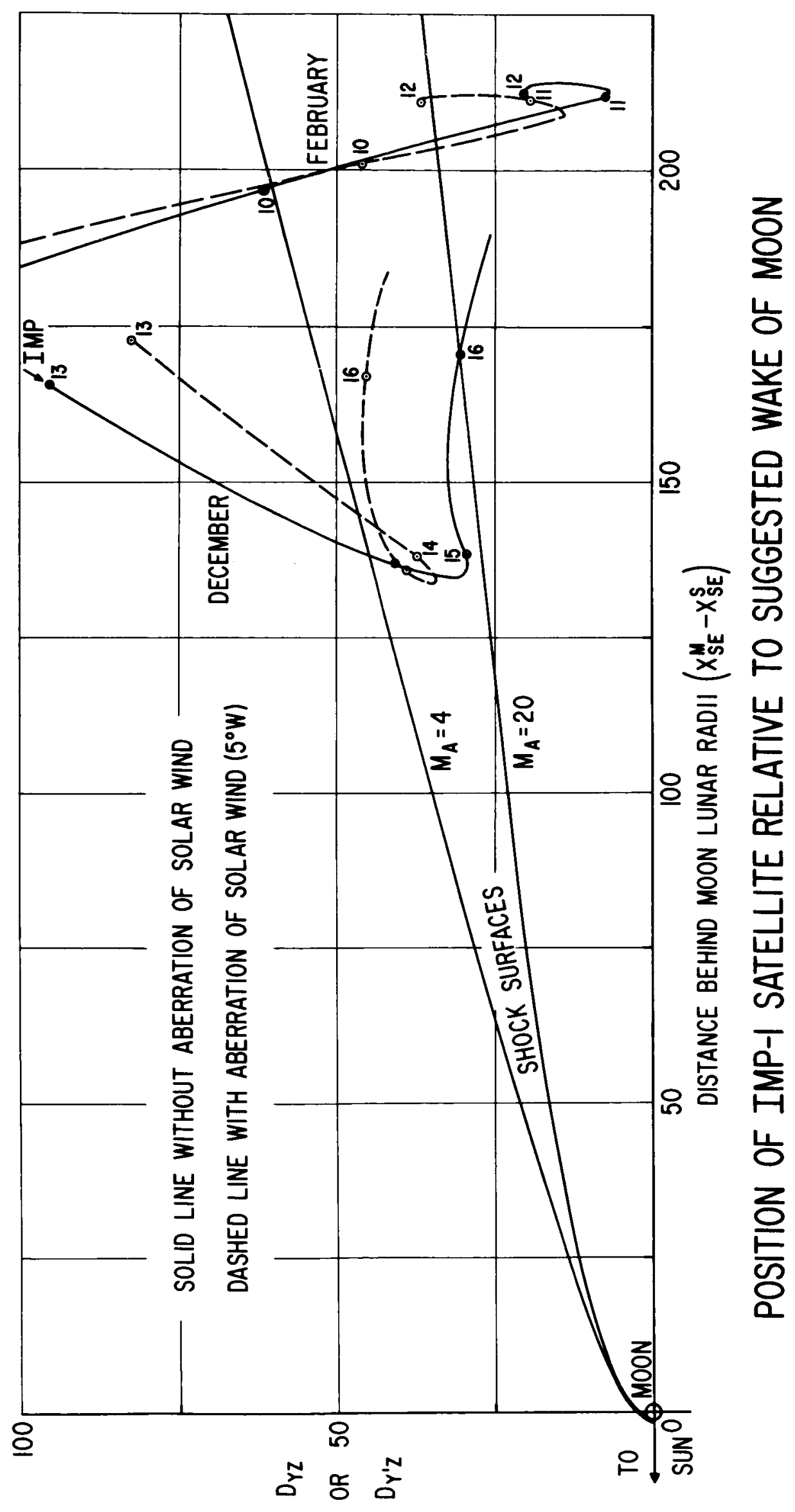

